\title{
Wave operators, similarity and dynamics for a class of Schrödinger operators with generic non-mixed interface conditions in $1 \mathrm{D}$.
}

\author{
Andrea Mantile*
}

\begin{abstract}
We consider a simple modification of the 1D-Laplacian where non-mixed interface conditions occur at the boundaries of a finite interval. It has recently been shown that Schrödinger operators having this form allow a new approach to the transverse quantum transport through resonant heterostructures. In this perspective, it is important to control the deformations effects introduced on the spectrum and on the time propagator by this class of non-selfadjont perturbations. In order to obtain uniform-in-time estimates of the perturbed semigroup, our strategy consists in constructing stationary waves operators allowing to intertwine the modified non-selfadjoint Schrödinger operator with a 'physical' Hamiltonian. For small values of a deformation parameter ' $\theta$ ', this yields a dynamical comparison between the two models showing that the distance between the corresponding semigroups is dominated by $|\theta|$ uniformly in time in the $L^{2}$-operator norm.
\end{abstract}

AMS Subject Classification: 81Q12, 47A40, 58J50

\section{Introduction.}

Schrödinger operators with non-mixed interface conditions have been recently considered in [12, by introducing the modified 1D Laplacian $\Delta_{\theta}$

$$
\left\{\begin{array}{l}
D\left(\Delta_{\theta}\right)=\left\{u \in H^{2}(\mathbb{R} \backslash\{a, b\}):\left[\begin{array}{l}
e^{-\frac{\theta}{2}} u\left(b^{+}\right)=u\left(b^{-}\right) ; e^{-\frac{3}{2} \theta} u^{\prime}\left(b^{+}\right)=u^{\prime}\left(b^{-}\right) \\
e^{-\frac{\theta}{2}} u\left(a^{-}\right)=u\left(a^{+}\right) ; e^{-\frac{3}{2} \theta} u^{\prime}\left(a^{-}\right)=u^{\prime}\left(a^{+}\right)
\end{array}\right\},\right. \\
\Delta_{\theta} u(x)=u^{\prime \prime}(x) \text { for } x \in \mathbb{R} \backslash\{a, b\}
\end{array}\right.
$$

where $u\left(x^{ \pm}\right)$respectively denote the right and left limit of the function $u$ in $x$. For all $\theta \in \mathbb{C} \backslash\{0\}$, the operator $\Delta_{\theta}$ describes a singularly perturbed Laplacian, with non-selfadjoint point interactions acting in the boundary points $\{a, b\}$. It is worthwhile to notice that the boundary conditions in (1.1) do not model an usual non-selfadjoint point interaction (that is $\delta$ or $\delta^{\prime}$ type).

The interest in quantum models arising from $\Delta_{\theta}$ stands upon the fact that a sharp exterior complex dilation, depending on $\theta=i \tau$ with $\tau>0$, maps $-i \Delta_{\theta}$ into the accretive operator: $-i e^{-2 \theta 1_{\mathbb{R} \backslash(a, b)}(x)} \Delta_{2 \theta}$, where $1_{D}$ denotes the characteristic function of the domain $D$ (e.g. in Lemma 3.1 in [12]). For a shortrange potential $\mathcal{V}$ (i.e.: $\mathcal{V} \in L^{1}$ ) compactly supported in $[a, b]$, the corresponding complex deformed Schrödinger operator

$$
\mathcal{H}_{\theta}(\mathcal{V}, \theta)=-e^{-2 \theta 1_{\mathbb{R} \backslash(a, b)}(x)} \Delta_{2 \theta}+\mathcal{V}, \quad \operatorname{supp} \mathcal{V}=[a, b],
$$

is the generator of a contraction semigroup and, in the case of time dependent potentials, uniform-in-time estimates hold for the dynamical system. According to the complex dilation technique (see [1, 2]) the quantum resonances of the undeformed operator $\mathcal{H}_{\theta}(\mathcal{V})$

$$
\mathcal{H}_{\theta}(\mathcal{V})=-\Delta_{\theta}+\mathcal{V}
$$

are detected by exterior complex dilations and identify with the spectral points of $\mathcal{H}_{\theta}(\mathcal{V}, \theta)$ in a suitable sector of the second Riemann sheet. Then, the adiabatic evolution problem for the resonant states

\footnotetext{
${ }^{*}$ Laboratoire de Mathématiques de Reims, EA4535 URCA, Fédération de Recherche ARC Mathématiques, FR 3399 CNRS.
} 
of $\mathcal{H}_{\theta}(\mathcal{V})$ rephrases, through an exterior complex dilation, as the adiabatic evolution problem for the corresponding eigenstates of $\mathcal{H}_{\theta}(\mathcal{V}, \theta)$. In this framework, accounting the contractivity property of the semigroup $e^{-i t \mathcal{H}_{\theta}(\mathcal{V}, \theta)}$ a 'standard' adiabatic theory can be developed (e.g. in [23]). This approach has been introduced in 12 where an adiabatic theorem is obtained for shape resonances in the regime of quantum wells in a semiclassical island. The purpose of this work is to justify the use of Hamiltonians of the type $\mathcal{H}_{\theta}(\mathcal{V})$ in the modelling of quantum systems.

The relevance of the artificial interface conditions (1.1), stands upon the fact that they are expected to introduce small errors, w.r.t. the selfadjoint case, controlled by $|\theta|$. The quantum dynamics generated by $\Delta_{\theta}$ has been considered in [12]. The explicit character of the model allows to obtain the asymptotic expansion

$$
e^{-i t \Delta_{\theta}}=e^{-i t \Delta}+\mathcal{R}(t, \theta)
$$

holding in a suitable neighbourhood: $|\theta|<\delta$ (see Proposition 2.2 in [12]). Here, the reminder $\mathcal{R}(t, \theta)$ is strongly continuous w.r.t. $t$ and $\theta$, exhibits the group property w.r.t. the time variable and is such that

$$
\sup _{t \in \mathbb{R}}\|\mathcal{R}(t, \theta)\|_{\mathcal{L}\left(L^{2}(\mathbb{R})\right)}=\mathcal{O}(|\theta|)
$$

Thus, for $\theta$ small enough, $\Delta_{\theta}$ generates a group, strongly continuous both w.r.t. $t$ and $\theta$, and allowing uniform-in-time estimates. In the perspective of modelling realistic physical situations through the modified Schrödinger operators $\mathcal{H}_{\theta}(\mathcal{V})$, an important step would consists in extending to this class of operators the expansion obtained in (1.4) for $\mathcal{H}_{\theta}(0)=-\Delta_{\theta}$. A possible approach considers $\mathcal{H}_{\theta}(\mathcal{V})$ as a (selfadjoint) perturbation of the modified Laplacian: $\mathcal{H}_{\theta}(0)$; it is worthwhile to notice that this would give a weaker result. For instance, implementing a Picard iteration on the Duhamel formula

$$
u_{t}(\theta)=e^{-i t \Delta_{\theta}} u_{0}+i \int_{0}^{t} e^{-i(t-s) \Delta_{\theta}} \mathcal{V} u_{s}(\theta) d s,
$$

and making use of the expansion (1.4), yields, in the case of a bounded potential $\mathcal{V} \in L^{\infty}$, the time dependent estimates

$$
\begin{gathered}
\left\|u_{t}(\theta)\right\|_{L^{2}(\mathbb{R})} \leq C_{1}\left\|u_{0}\right\|_{L^{2}(\mathbb{R})} e^{C_{2}\|\mathcal{V}\|_{L^{\infty}(\mathbb{R})} t} \\
\left\|u_{t}(\theta)-u_{t}(0)\right\|_{L^{2}(\mathbb{R})} \leq C_{3}|\theta|\left\|u_{0}\right\|_{L^{2}(\mathbb{R})} t e^{C_{4}\|\mathcal{V}\|_{L^{\infty}(\mathbb{R})} t}
\end{gathered}
$$

where $C_{i}, i=1, . .4$, are suitable positive constants. It follows that, for an initial state $u_{0}$, the corresponding mild solution to the quantum evolution problem, $u_{t}(\theta)$, is Lipschitz continuous w.r.t. $\theta$, with a Lipschitz constant bounded by an exponentially increasing function of time. As an aside, we notice that the estimate (1.7) may also be obtained as a consequence of the Hille-Yoshida-Phillips Theorem, by using the second resolvent formula for $\left(\mathcal{H}_{\theta}(\mathcal{V})-z\right)^{-1}$ and resolvent estimates for $\left(\mathcal{H}_{\theta}(0)-z\right)^{-1}$ arising from (1.4).

The relation (1.8) yields a finite-time control, depending on $\|\mathcal{V}\|_{L^{\infty}(\mathbb{R})}$, of the error introduced on the quantum evolution by the interface conditions. However, when $\mathcal{V}$ describes the (possibly non-linear) interactions involving charge carriers in resonant heterostructures, its norm $\|\mathcal{V}\|_{L^{\infty}(\mathbb{R})}$ is expected to be small compared to the energy of the particles, while the quantum evolution of relevant observables is characterized by a long time scale, corresponding to the inverse of the imaginary part of the shape resonances (examples of this mechanism are exhibited in 25 and 13]). In this framework, the use of modified Hamiltonians of the type $\mathcal{H}_{\theta}(\mathcal{V})$ would be justified by a stronger uniform-in-time estimate for the error $\left\|u_{t}(\theta)-u_{t}(0)\right\|_{L^{2}(\mathbb{R})}$ as $\theta \rightarrow 0$.

Adopting a different approach, in what follows the operator $\mathcal{H}_{\theta}(\mathcal{V})$ is considered as a non-selfadjoint perturbation of the selfadjoint Hamiltonian $\mathcal{H}_{0}(\mathcal{V})$. Non-selfadjoint perturbations of the type $T(x)=$ $T+x A B^{*}$ have been studied in [17] where, under smoothness assumptions on $A$ and $B$, the 'stationary' wave operators for the couple $\{T(x), T\}$ are given and the corresponding similarity between $T$ and $T(x)$ is exploited to define the dynamics generated by $-i T(x)$. This strategy is adapted here to the case where $T=\mathcal{H}_{0}(\mathcal{V})$, while the perturbation is determined by generic, non-mixed, interface conditions occurring at the boundaries of the potential's support. This is a larger class of operators, parametrized by a couple of complex, which includes both the cases of $\mathcal{H}_{\theta}(\mathcal{V})$ and $\left(\mathcal{H}_{\theta}(\mathcal{V})\right)^{*}$. From an accurate resolvent analysis and explicit generalized eigenfunctions formulas, we deduce, in this extended framework, a small- $\theta$ expansion of the 'stationary wave operators'. Then the quantum evolution group generated by 
$-i \mathcal{H}_{\theta}(\mathcal{V})$ is determined by conjugation from $e^{-i t \mathcal{H}_{0}(\mathcal{V})}$ and an uniform-in-time estimate for the 'distance' between the two dynamics is obtained (see Theorem 1.1 below).

Similarity transformations, from non-selfadjoint to similar selfadjoint operators, have been recently studied in [18, where the authors focus on the particular case of 1D Schrödinger operators defined with non-selfadjoint Robin-type conditions occurring at the boundary of an interval. In the case of parity and time-reversal symmetry $(\mathcal{P} \mathcal{T}$-symmetry), the similarity of this model with a selfadjoint Hamiltonian is derived. It is worth noticing that, when $\theta \in i \mathbb{R}$, the modified Laplacian $\Delta_{\theta}$ actually exhibits the $\mathcal{P} \mathcal{T}$-symmetry (once the parity is defined with respect to the point $(a+b) / 2$ ). However, the models introduced in the next sections are generically not $\mathcal{P} \mathcal{T}$-symmetric (see the definition (1.11) below).

\subsection{Schrödinger operators with non-mixed interface conditions.}

We consider the family of modified Schrödinger operators $Q_{\theta_{1}, \theta_{2}}(\mathcal{V})$, depending on a couple of complex parameters, $\left(\theta_{1}, \theta_{2}\right) \in \mathbb{C}^{2}$, and on a selfadjoint short-range potential, compactly supported over the interval $[a, b] \subset \mathbb{R}$

$$
\mathcal{V} \in L^{1}(\mathbb{R}, \mathbb{R}), \quad \operatorname{supp} \mathcal{V}=[a, b] .
$$

The parameters $\theta_{1}$ and $\theta_{2}$ fix the interface conditions,

$$
\begin{cases}e^{-\frac{\theta_{1}}{2}} u\left(b^{+}\right)=u\left(b^{-}\right), & e^{-\frac{\theta_{2}}{2}} u^{\prime}\left(b^{+}\right)=u^{\prime}\left(b^{-}\right), \\ e^{-\frac{\theta_{1}}{2}} u\left(a^{-}\right)=u\left(a^{+}\right), & e^{-\frac{\theta_{2}}{2}} u^{\prime}\left(a^{-}\right)=u\left(a^{+}\right),\end{cases}
$$

occurring at the boundary of the potential's support and $Q_{\theta_{1}, \theta_{2}}(\mathcal{V})$ is defined as follows

$$
Q_{\theta_{1}, \theta_{2}}(\mathcal{V}):\left\{\begin{array}{l}
D\left(Q_{\theta_{1}, \theta_{2}}(\mathcal{V})\right)=\left\{u \in H^{2}(\mathbb{R} \backslash\{a, b\}) \mid \text { (1.10) holds }\right\}, \\
\left(Q_{\theta_{1}, \theta_{2}}(\mathcal{V}) u\right)(x)=-u^{\prime \prime}(x)+\mathcal{V}(x) u(x), \quad x \in \mathbb{R} \backslash\{a, b\} .
\end{array}\right.
$$

The set $\left\{Q_{\theta_{1}, \theta_{2}}(\mathcal{V}),\left(\theta_{1}, \theta_{2}\right) \in \mathbb{C}^{2}\right\}$ is closed w.r.t. the adjoint operation: a direct computation shows that

$$
\left(Q_{\theta_{1}, \theta_{2}}(\mathcal{V})\right)^{*}=Q_{-\theta_{2}^{*},-\theta_{1}^{*}}(\mathcal{V}) .
$$

The subset of selfadjoint operators in this class is identified by the conditions: for $\theta_{j}=r_{j} e^{i \varphi_{j}}, j=1,2$,

$$
\left\{\begin{array}{l}
\varphi_{1}+\varphi_{2}=\pi+2 \pi k, \quad k \in \mathbb{Z} \\
r_{1}=r_{2}
\end{array}\right.
$$

When (1.13) are not satisfied, the corresponding operator $Q_{\theta_{1}, \theta_{2}}(\mathcal{V})$ is neither selfadjoint nor symmetric, since in this case: $Q_{\theta_{1}, \theta_{2}}(\mathcal{V}) \not \subset\left(Q_{\theta_{1}, \theta_{2}}(\mathcal{V})\right)^{*}$.

For each couple $\left\{\theta_{1}, \theta_{2}\right\}, Q_{\theta_{1}, \theta_{2}}(\mathcal{V})$ identifies with a, possibly non-selfadjoint, extension of the Hermitian operator $Q^{0}(\mathcal{V})$

$$
D\left(Q^{0}(\mathcal{V})\right)=\left\{u \in H^{2}(\mathbb{R}) \mid u(\alpha)=u^{\prime}(\alpha)=0, \alpha=a, b\right\},
$$

and defines an explicitly solvable model w.r.t. the selfadjoint Hamiltonian $Q_{0,0}(\mathcal{V})$. Non-selfadjoint models arising from proper extensions of Hermitian operators with gaps have been already considered in literature, for instance in [1], 30] (see also [20]-22] and [9] for the general case of adjoint pairs of operators). In these works, the formalism of boundary triples (e.g. in [19] for adjoint pairs) is adopted; this leads to Krein-like formulas expressing the resolvent of an extended operator in terms of the resolvent of a 'reference' extension plus a finite rank part depending on the Weyl function of the triple. In Section 2 we give Krein-like formulas for the difference $\left(Q_{\theta_{1}, \theta_{2}}(\mathcal{V})-z\right)^{-1}-\left(Q_{0,0}(\mathcal{V})-z\right)^{-1}$. Exploiting this framework, we show that $Q_{\theta_{1}, \theta_{2}}(\mathcal{V})$ is an analytic family in the sense of Kato both w.r.t. the variables $\theta_{1}$ and $\theta_{2}$ and study its spectral profile depending on $\mathcal{V}$. The result is exposed in the Proposition 2.5] in particular, for a defined positive $\mathcal{V}$, we obtain: $\sigma\left(Q_{\theta_{1}, \theta_{2}}(\mathcal{V})\right)=\sigma_{a c}\left(Q_{0,0}(\mathcal{V})\right)=\mathbb{R}_{+}$, provided that $\theta_{1}$ and $\theta_{2}$ are small enough.

Under the same assumptions, in Section 3 , a family of intertwining operators $\mathcal{W}_{\theta_{1}, \theta_{2}}$ for the couple $\left\{Q_{\theta_{1}, \theta_{2}}(\mathcal{V}), Q_{0,0}(\mathcal{V})\right\}$ is introduced as the analogous of the usual stationary wave operators in selfadjoint frameworks. Using the eigenfunctions expansion obtained in Subsection 2.3. we get a small- $\theta_{i}$ expansion of $\mathcal{W}_{\theta_{1}, \theta_{2}}$ allowing to define the quantum evolution group $e^{-i Q_{\theta_{1}, \theta_{2}}(\mathcal{V})}$ from $e^{-i Q_{0,0}(\mathcal{V})}$ by conjugation. Then, we develop a quantitative comparison showing that $e^{-i Q_{\theta_{1}, \theta_{2}}(\mathcal{V})}-e^{-i Q_{0,0}(\mathcal{V})}$ is controlled by $\left|\theta_{i}\right|$, 
$i=1,2$, uniformly in time, in the $L^{2}$-operator norm. The result is presented in the following theorem and the proof is given in Subsection 3.1 It can be adapted to the particular case of $\mathcal{H}_{\theta}(\mathcal{V})$, by noticing that: $\mathcal{H}_{\theta}(\mathcal{V})=Q_{\theta, 3 \theta}(\mathcal{V})$

Theorem 1.1 Let $\mathcal{V}$ fulfills the conditions (1.9),

$$
\langle u, \mathcal{V} u\rangle_{L^{2}((a, b))}>0 \quad \forall u \in L^{2}((a, b)),
$$

and assume $\left|\theta_{j}\right|<\delta, j=1,2$, with $\delta>0$ small enough. Then $-i Q_{\theta_{1}, \theta_{2}}(\mathcal{V})$ generates a strongly continuous group of bounded operators on $L^{2}(\mathbb{R}),\left\{e^{-i t Q_{\theta_{1}, \theta_{2}}(\mathcal{V})}\right\}_{t \in \mathbb{R}}$. For a fixed $t, e^{-i t Q_{\theta_{1}, \theta_{2}}(\mathcal{V})}$ defines an analytic family of bounded operators w.r.t. $\left(\theta_{1}, \theta_{2}\right)$ and the expansion

$$
e^{-i t Q_{\theta_{1}, \theta_{2}}(\mathcal{V})}=e^{-i t Q_{0,0}(\mathcal{V})}+\mathcal{R}\left(t, \theta_{1}, \theta_{2}\right)
$$

holds with an uniformly bounded in time reminder s.t.

$$
\sup _{t \in \mathbb{R}}\left\|\mathcal{R}\left(t, \theta_{1}, \theta_{2}\right)\right\|_{\mathcal{L}\left(L^{2}(\mathbb{R})\right)}=\mathcal{O}\left(\theta_{1}\right)+\mathcal{O}\left(\theta_{2}\right) .
$$

In the Subsection 3.2 the pair $\left\{Q_{\theta_{1}, \theta_{2}}(\mathcal{V}), Q_{0,0}(\mathcal{V})\right\}$ is considered as a scattering system and we investigate the existence of non-stationary wave operators. Under the assumptions of the Theorem 1.1. it is shown that $\mathcal{W}_{\theta_{1}, \theta_{2}}$ coincides with a 'physical' wave operator, according to the time dependent definition (Lemma 3.2). Although exploited in our analysis, the small perturbations condition does not seem to be necessary in the proof of this result: a possible strategy for its extension to the case where $\left\{\theta_{1}, \theta_{2}\right\}$ are not small is finally mentioned.

Further perspectives of this work, concerning the regime of quantum wells in a semiclassical island, are discussed in the Section 4

\subsection{Notation}

In what follows, we make use of a generalization of the Landau notation, $\mathcal{O}(\cdot)$, defined according to:

Definition 1.2 Let be $X$ a metric space and $f, g: X \rightarrow \mathbb{C}$. Then $f=\mathcal{O}(g) \stackrel{\text { def }}{\Longleftrightarrow} \forall x \in X$ it holds: $f(x)=p(x) g(x)$, being $p$ a bounded map $X \rightarrow \mathbb{C}$.

The next notation are also adopted

$1_{\Omega}(\cdot)$ is the characteristic function of a domain $\Omega$.

$\mathcal{B}_{\delta}(p)$ is the open ball of radius $\delta$ centered in a point $p \in \mathbb{C}$.

$\mathcal{C}_{x}^{k}(U)$ is the set of $\mathcal{C}^{k}$-continuous functions w.r.t. $x \in U \subseteq \mathbb{R}$.

$\mathcal{H}_{z}(D)$ is the set of holomorphic functions w.r.t. $z \in D \subseteq \mathbb{C}$.

$\partial_{j} f\left(x_{1}, \ldots x_{n}\right), j \in\{1, . . n\}$, denotes the derivative of $f$ w.r.t. the variable $x_{j}$.

$S_{\eta}$ denotes the complex half-plane: $S_{\eta}=\{z \in \mathbb{C} \mid \operatorname{Im} z>-\eta\}$. In particular, $\mathbb{C}^{+}$coincides with $S_{0}$.

The notation ' $\lesssim$ ', appearing in some of the proofs, denotes the inequality: ' $\leq C$ ' being $C$ a suitable positive constant.

\section{Boundary triples and Krein-like resolvent formulas.}

Point perturbation models, as $Q_{\theta_{1}, \theta_{2}}(\mathcal{V})$, can be described as restrictions of a larger operator through linear relations on an Hilbert space. Let introduce $Q(\mathcal{V})$

$$
\left\{\begin{array}{l}
D(Q(\mathcal{V}))=H^{2}(\mathbb{R} \backslash\{a, b\}), \\
(Q(\mathcal{V}) u)(x)=-u^{\prime \prime}(x)+\mathcal{V}(x) u(x) \quad \text { for } x \in \mathbb{R} \backslash\{a, b\},
\end{array}\right.
$$

with $\mathcal{V}$ defined according to (1.9), and let $Q^{0}(\mathcal{V})$ be such that: $\left(Q^{0}(\mathcal{V})\right)^{*}=Q(\mathcal{V})$. Explicitly, $Q^{0}(\mathcal{V})$ identifies with the symmetric restriction of $Q(\mathcal{V})$ to the domain

$$
D\left(Q^{0}(\mathcal{V})\right)=\left\{u \in D(Q(\mathcal{V})) \mid u(\alpha)=u^{\prime}(\alpha)=0 \forall \alpha \in\{a, b\}\right\} .
$$


The related defect spaces, $\mathcal{N}_{z}=\operatorname{ker}(Q(\mathcal{V})-z)$, are 4-dimensional subspaces of $D(Q(\mathcal{V}))$ generated, for $z \in \mathbb{C} \backslash \mathbb{R}$, by the independent solutions to the problem

$$
\left\{\begin{array}{l}
\left(-\partial_{x}^{2}+\mathcal{V}-z\right) u(x)=0, \quad x \in \mathbb{R} \backslash\{a, b\} \\
u \in D(Q(\mathcal{V}))
\end{array}\right.
$$

A boundary triple $\left\{\mathbb{C}^{4}, \Gamma_{0}, \Gamma_{1}\right\}$ for $Q(\mathcal{V})$ is defined with two linear boundary maps $\Gamma_{i=1,2}: D(Q(\mathcal{V})) \rightarrow \mathbb{C}^{4}$ fulfilling, for any $\psi, \varphi \in D(Q(\mathcal{V}))$, the equation

$$
\langle\psi, Q(\mathcal{V}) \varphi\rangle_{L^{2}(\mathbb{R})}-\langle Q(\mathcal{V}) \psi, \varphi\rangle_{L^{2}(\mathbb{R})}=\left\langle\Gamma_{0} \psi, \Gamma_{1} \varphi\right\rangle_{\mathbb{C}^{4}}-\left\langle\Gamma_{1} \psi, \Gamma_{0} \varphi\right\rangle_{\mathbb{C}^{4}}
$$

and such that the transformation $\left(\Gamma_{0}, \Gamma_{1}\right): D(Q(\mathcal{V})) \rightarrow \mathbb{C}^{4} \times \mathbb{C}^{4}$ is surjective. A proper extension $Q_{\text {ext }}$ of $Q^{0}(\mathcal{V})$ is called almost solvable if there exists a boundary triple $\left\{\mathbb{C}^{4}, \Gamma_{0}, \Gamma_{1}\right\}$ and a matrix $M \in \mathbb{C}^{4,4}$ such that it coincides with the restriction of $Q(\mathcal{V})$ to the domain: $\left\{u \in D(Q(\mathcal{V})) \mid M \Gamma_{0} u=\Gamma_{1} u\right\}$. Using the notation $Q_{e x t}=Q_{M}(\mathcal{V})$, the characterization

$$
Q^{0}(\mathcal{V}) \subset Q_{M}(\mathcal{V}) \subset Q(\mathcal{V}), \quad\left(Q_{M}(\mathcal{V})\right)^{*}=Q_{M^{*}}(\mathcal{V})
$$

holds (e.g. [30, Theorem 1.1). In what follows, $\tilde{Q}(\mathcal{V})$ denotes the particular restriction of $Q(\mathcal{V})$ associated with the conditions: $\Gamma_{0} u=0$, i.e.

$$
D(\tilde{Q}(\mathcal{V}))=\left\{u \in D(Q(\mathcal{V})), \Gamma_{0} u=0\right\}
$$

According to the relation (2.4), $\tilde{Q}(\mathcal{V})$ is selfadjoint and $\mathbb{C} \backslash \mathbb{R} \subset \rho(\tilde{Q}(\mathcal{V}))$. Let, $\gamma(z, \mathcal{V})$ and $q(z, \mathcal{V})$ be the linear maps defined by

$$
\gamma(z, \mathcal{V})=\left(\left.\Gamma_{0}\right|_{\mathcal{N}_{z}}\right)^{-1}, \quad q(z, \mathcal{V})=\Gamma_{1} \circ \gamma(z, \mathcal{V}), \quad z \in \rho(\tilde{Q}(\mathcal{V}))
$$

where $\left.\Gamma_{0}\right|_{\mathcal{N}_{z}}$ is the restriction of $\Gamma_{0}$ to $\mathcal{N}_{z}$. These define holomorphic families of bounded operators in $\mathcal{L}\left(\mathbb{C}^{4}, L^{2}(\mathbb{R})\right)$ and $\mathcal{L}\left(\mathbb{C}^{4}, \mathbb{C}^{4}\right)$ (e.g. in [1] and [10]). The maps $\gamma(\cdot, z, \mathcal{V})$ and $q(z, \mathcal{V})$ are respectively referred to as the Gamma field and the Weyl function associated with the triple $\left\{\mathbb{C}^{4}, \Gamma_{0}, \Gamma_{1}\right\}$. With this formalism, a resolvent formula expresses the difference $\left(Q_{M}(\mathcal{V})-z\right)^{-1}-(\tilde{Q}(\mathcal{V})-z)^{-1}$ in terms of finite rank operator with range $\mathcal{N}_{z}$

$$
\left(Q_{M}(\mathcal{V})-z\right)^{-1}-(\tilde{Q}(\mathcal{V})-z)^{-1}=\gamma(z, \mathcal{V})(M-q(z, \mathcal{V}))^{-1} \gamma^{*}(\bar{z}, \mathcal{V}), \quad z \in \rho\left(Q_{M}(\mathcal{V})\right) \cap \rho(\tilde{Q}(\mathcal{V}))
$$

(e.g. in [30], Theorem 1.2). In many situations, the interface conditions occurring in the points $\{a, b\}$ can also be represented in the form: $A \Gamma_{0} u=B \Gamma_{1} u$, where $A, B \in \mathbb{C}^{4,4}$. We denote with $Q_{A, B}(\mathcal{V})$ the corresponding restriction

$$
\left\{\begin{array}{l}
D\left(Q_{A, B}(\mathcal{V})\right)=\left\{u \in D(Q(\mathcal{V})) \mid A \Gamma_{0} u=B \Gamma_{1} u\right\} \\
Q_{A, B}(\mathcal{V}) u=Q(\mathcal{V}) u
\end{array} .\right.
$$

With this parametrization, we have: $\tilde{Q}(\mathcal{V})=Q_{1,0}(\mathcal{V})$, while the resolvent's formula rephrases as

$$
\left(Q_{M}(\mathcal{V})-z\right)^{-1}-(\tilde{Q}(\mathcal{V})-z)^{-1}=-\gamma(z, \mathcal{V})\left[(B q(z, \mathcal{V})-A)^{-1} B\right] \gamma^{*}(\bar{z}, \mathcal{V}), \quad z \in \rho\left(Q_{M}(\mathcal{V})\right)
$$

In the perspective of a comparison between the quantum models arising from $Q_{\theta_{1}, \theta_{2}}(\mathcal{V})$ and $Q_{0,0}(\mathcal{V})$ a natural choice is

$$
\Gamma_{0} u=\left(\begin{array}{c}
u^{\prime}\left(b^{-}\right)-u^{\prime}\left(b^{+}\right) \\
u\left(b^{+}\right)-u\left(b^{-}\right) \\
u^{\prime}\left(a^{-}\right)-u^{\prime}\left(a^{+}\right) \\
u\left(a^{+}\right)-u\left(a^{-}\right)
\end{array}\right), \quad \Gamma_{1} u=\frac{1}{2}\left(\begin{array}{c}
u\left(b^{+}\right)+u\left(b^{-}\right) \\
u^{\prime}\left(b^{+}\right)+u^{\prime}\left(b^{-}\right) \\
u\left(a^{+}\right)+u\left(a^{-}\right) \\
u^{\prime}\left(a^{+}\right)+u^{\prime}\left(a^{-}\right)
\end{array}\right),
$$


which leads to: $\tilde{Q}(\mathcal{V})=Q_{0,0}(\mathcal{V})$. According to the definitions (1.11) and (2.1), the operator $Q_{\theta_{1}, \theta_{2}}(\mathcal{V})$ identifies with the restriction of $Q(\mathcal{V})$ parametrized by the $\mathbb{C}^{4,4}$-block-diagonal matrices

$$
A_{\theta_{1}, \theta_{2}}=\left(\begin{array}{ll}
a\left(\theta_{1}, \theta_{2}\right) & \\
& a\left(-\theta_{1},-\theta_{2}\right)
\end{array}\right), \quad B_{\theta_{1}, \theta_{2}}=\left(\begin{array}{ll}
b\left(\theta_{1}, \theta_{2}\right) & \\
& b\left(-\theta_{1},-\theta_{2}\right)
\end{array}\right),
$$

defined with

$$
a\left(\theta_{1}, \theta_{2}\right)=\left(\begin{array}{cc}
1+e^{\frac{\theta_{2}}{2}} & 0 \\
0 & 1+e^{\frac{\theta_{1}}{2}}
\end{array}\right), \quad b\left(\theta_{1}, \theta_{2}\right)=2\left(\begin{array}{cc}
0 & 1-e^{\frac{\theta_{2}}{2}} \\
e^{\frac{\theta_{1}}{2}}-1 & 0
\end{array}\right) .
$$

Using (2.11) and (2.13)-(2.13), the linear relations (1.10) rephrase as

$$
A_{\theta_{1}, \theta_{2}} \Gamma_{0} u=B_{\theta_{1}, \theta_{2}} \Gamma_{1} u,
$$

which leads to the equivalent definition

$$
Q_{\theta_{1}, \theta_{2}}(\mathcal{V}):\left\{\begin{array}{l}
\mathcal{D}\left(Q_{\theta_{1}, \theta_{2}}(\mathcal{V})\right)=\left\{u \in D(Q(\mathcal{V})) \mid A_{\theta_{1}, \theta_{2}} \Gamma_{0} u=B_{\theta_{1}, \theta_{2}} \Gamma_{1} u\right\}, \\
Q_{\theta_{1}, \theta_{2}}(\mathcal{V}) u=Q(\mathcal{V}) u .
\end{array}\right.
$$

In this framework, the relation (2.10) explicitly writes as

$$
\left(Q_{\theta_{1}, \theta_{2}}(\mathcal{V})-z\right)^{-1}=\left(Q_{0,0}(\mathcal{V})-z\right)^{-1}-\sum_{i, j=1}^{4}\left[\left(B_{\theta_{1}, \theta_{2}} q(z, \mathcal{V})-A_{\theta_{1}, \theta_{2}}\right)^{-1} B_{\theta_{1}, \theta_{2}}\right]_{i j}\left\langle\gamma\left(e_{j}, \bar{z}, \mathcal{V}\right), \cdot\right\rangle_{L^{2}(\mathbb{R})} \gamma\left(e_{i}, z, \mathcal{V}\right)
$$

where $\left\{e_{i}\right\}_{i=1}^{4}$ is the standard basis in $\mathbb{C}^{4}$, while $\gamma(v, z, \mathcal{V})$ denotes the action of $\gamma(z, \mathcal{V})$ on the vector $v$. The corresponding integral kernel, $\mathcal{G}_{\theta_{1}, \theta_{2}}^{z}(x, y)$, is

$$
\mathcal{G}_{\theta_{1}, \theta_{2}}^{z}(x, y)=\mathcal{G}_{0,0}^{z}(x, y)-\sum_{i, j=1}^{4}\left[\left(B_{\theta_{1}, \theta_{2}} q(z, \mathcal{V})-A_{\theta_{1}, \theta_{2}}\right)^{-1} B_{\theta_{1}, \theta_{2}}\right]_{i j} \gamma\left(e_{j}, y, z, \mathcal{V}\right) \gamma\left(e_{i}, x, z, \mathcal{V}\right)
$$

\subsection{The Jost's solutions.}

In order to obtain explicit representations of the operators $\gamma(\cdot, z, \mathcal{V})$ and $q(z, \mathcal{V})$ appearing at the r.h.s. of (2.16), it is necessary to define a particular basis of the defect spaces $\mathcal{N}_{z}$. A possible choice is given in terms of the Green's function of the operator $\left(Q_{0,0}(\mathcal{V})-z\right)$ and of their derivatives. This motivates the forthcoming analysis, where the properties of the functions in $\mathcal{N}_{z}$ are investigated by using the Jost's solutions associated with $Q_{0,0}(\mathcal{V})$. Our aim is to provide with explicit low and high energy asymptotic in the case of compactly supported and defined positive potentials. We follow a standard approach adapting arguments from one dimensional scattering to this particular case. Detailed computations, for selfadjoint one-dimensional Schrödinger operators with generic short range potentials, are presented in 31.

Consider the problem

$$
\left(-\partial_{x}^{2}+\mathcal{V}\right) u=\zeta^{2} u, \quad \text { for } x \in \mathbb{R} \text { and } \zeta \in \mathbb{C}^{+} .
$$

The Jost solutions to (2.18), $\chi_{ \pm}$, are respectively defined by the exterior conditions

$$
\left.\chi_{+}\right|_{x>b}=e^{i \zeta x},\left.\quad \chi_{-}\right|_{x<a}=e^{-i \zeta x} .
$$

The next proposition resumes some properties of the functions $\chi_{ \pm}$in the case of compactly supported potentials.

Proposition 2.1 Let $\mathcal{V}$ be defined according to (1.9). The solutions $\chi_{ \pm}$to the problem (2.18)-(2.19) belong to $\mathcal{C}_{x}^{1}\left(\mathbb{R}, \mathcal{H}_{\zeta}\left(\mathbb{C}^{+}\right)\right)$having continuous extension to the real axis. For $\zeta \in \overline{\mathbb{C}^{+}}$, the relations

$$
\chi_{ \pm}(x, \zeta)=e^{ \pm i \zeta x} \mathcal{O}(1), \quad \partial_{x} \chi_{ \pm}(x, \zeta)=e^{ \pm i \zeta x} \mathcal{O}(1+|\zeta|),
$$

hold with $\mathcal{O}(\cdot)$ referred to the metric space $\mathbb{R} \times \overline{\mathbb{C}^{+}}$. 
The proof follows, with slightly modifications, the one given in [31] in the case of 1D short-range potentials: an integral setting for (2.18) and explicit estimates for the corresponding integral kernel are used to discuss the convergence of the solution developed as a Picard series. To this aim, we need the following simple Lemma.

Lemma 2.2 Let $\mathcal{V}$ be defined according to [1.9) and $F(x)=\left|\int_{x}^{x_{0}}\right| \mathcal{V}(t)|d t|$, with $x_{0} \neq x$. If $f$ is continuous and such that: $|f(x)| \leq \frac{F^{n}(x)}{n !}$ for $n \in \mathbb{N}$, then it results

$$
\left|\int_{x}^{x_{0}} f(t) \mathcal{V}(t) d t\right| \leq \frac{F^{n+1}(x)}{(n+1) !} .
$$

Proof. For $x_{0}>x$ we have $F(x)=\int_{x}^{x_{0}}|\mathcal{V}(t)| d t$, we get: $\partial_{x} F(x)=-|\mathcal{V}(x)|$. Then

$$
\left|\int_{x}^{x_{0}} f(t) \mathcal{V}(t) d t\right| \leq-\int_{x}^{x_{0}}|f(t)| \partial_{t} F(t) d t \leq-\int_{x}^{x_{0}} \frac{F^{n}(x)}{n !} \partial_{t} F(t) d t=-\int_{x}^{x_{0}} \frac{\partial_{t} F^{n+1}(x)}{(n+1) !} d t .
$$

For $x_{0}<x$, we have $F(x)=\int_{x_{0}}^{x}|\mathcal{V}(t)| d t$ and $\partial_{x} F(x)=|\mathcal{V}(x)|$. Then

$$
\left|\int_{x_{0}}^{x} f(t) \mathcal{V}(t) d t\right| \leq \int_{x_{0}}^{x}|f(t)| \partial_{t} F(t) d t \leq \int_{x_{0}}^{x} \frac{F^{n}(x)}{n !} \partial_{t} F(t) d t=\int_{x_{0}}^{x} \frac{\partial_{t} F^{n+1}(x)}{(n+1) !} d t .
$$

Both the above relations imply (2.21) since, by definition, $F\left(x_{0}\right)=0$.

Proof of the Proposition 2.1. Here we focus on the case of $\chi_{+}$, while the problem for $\chi_{-}$can be analyzed similarly. Using the integral kernel: $-(\zeta)^{-1} \sin \zeta(t-x)$, the equation (2.18) rephrases into the equivalent integral form

$$
u(x, \zeta)=u_{0}(x, \zeta)-\int_{x_{0}}^{x} \frac{\sin \zeta(t-x)}{\zeta} \mathcal{V}(t) u(t, \zeta) d t
$$

In order to account for the conditions (2.19), we replace in (2.22): $x_{0}=b, x<b$ and $u_{0}=e^{i \zeta x}$. Then, introducing the rescaled functions: $b_{+}=e^{-i \zeta x} \chi_{+}$and

$$
\mathcal{K}_{+}(t, x, \zeta)=-e^{i \zeta(t-x)} \frac{\sin \zeta(t-x)}{\zeta},
$$

we get the equation

$$
b_{+}(x, \zeta)=1-\int_{x}^{b} \mathcal{K}_{+}(t, x, \zeta) \mathcal{V}(t) b_{+}(t, \zeta) d t, \quad \text { for } x<b,
$$

while, for $x>b$ one has: $b_{+}=1$. The corresponding solution formally writes as a Picard series: $b_{+}=\sum_{n=0}^{+\infty} b_{+, n}$ whose terms are defined according to

$$
b_{+, 0}=1, \quad b_{+, n}(x, \zeta)=-\int_{x}^{b} \mathcal{K}_{+}(t, x, \zeta) \mathcal{V}(t) b_{+, n-1}(t, \zeta) d t, \quad n \in \mathbb{N}^{*}, \quad x<b .
$$

Let $\eta>0$ and introduce the auxiliary domain $S_{\eta}$ (see the definition given in the subsection 1.2). The rescaled kernel is a smooth map of $t$ and $x$ with values in $\mathcal{H}_{\zeta}\left(S_{\eta}\right)$; then, using (2.25), an induction over $n$ leads to: $b_{+, n} \in \mathcal{C}_{x}^{1}\left((-\infty, b), \mathcal{H}_{\zeta}\left(S_{\eta}\right)\right)$. Next, the convergence of the sum $\sum_{n=0}^{+\infty} b_{+, n}$ is considered, at first, in the case of a bounded interval $x \in(c, b)$, then in the whole interval $(-\infty, b)$. Finally, the low and high-energy behaviour of $\chi_{+}$are investigated to obtain the relations in (2.20). In what follows, we assume: $\eta>0, c<a$ and use the notation $U_{\eta, c}=(c, b) \times S_{\eta}$; a direct computation yields

$$
1_{(x, b)}(t) 1_{U_{\eta, c}}(x, \zeta) \mathcal{K}_{+}(t, x, \zeta)=\mathcal{O}\left(\frac{1}{1+|\zeta|}\right) ; \quad 1_{(x, b)}(t) 1_{U_{\eta, c}}(x, \zeta) \partial_{x} \mathcal{K}_{+}(t, x, \zeta)=\mathcal{O}(1)
$$

where the symbols $\mathcal{O}(\cdot)$, introduced in the Definition 1.2, here refer to the metric space: $(c, b)^{2} \times S_{\eta}$. According to (2.26), a positive constant $C_{a, b, c, \eta}$, possibly depending on the data, exists such that

$$
\sup _{\substack{\{x, \zeta\} \in U_{c, \eta} \\ t \in(x, b)}}\left|\mathcal{K}_{+}(t, x, \zeta)\right|<C_{a, b, c, \eta}
$$


Let introduce the rescaled potential: $\tilde{\mathcal{V}}(x)=C_{a, b, c, \eta} \mathcal{V}(x)$ and the function $F(x)=\int_{x}^{b}|\tilde{\mathcal{V}}(t)| d t$. As a consequence of Lemma 2.2 we have:

$\left|1_{U_{\eta, c}} b_{+, n+1}\right| \leq 1_{U_{\eta, c}} \frac{F^{n+1}}{(n+1) !}$. Since $\|F\|_{L^{\infty}(c, b)}=C_{a, b, c, \eta}\|\mathcal{V}\|_{L^{1}(a, b)}$, this yields the estimate

$$
\sup _{\{x, \zeta\} \in U_{c}^{+}}\left|1_{U_{\eta, c}} b_{+, n+1}\right| \leq \frac{C_{a, b, c, \eta}^{n+1}\|\mathcal{V}\|_{L^{1}(a, b)}^{n+1}}{(n+1) !}
$$

and the Picard series uniformly converges to $b_{+} \in \mathcal{C}_{x}^{0}\left((c, b), \mathcal{H}_{\zeta}\left(S_{\eta}\right)\right)$. In particular, (2.28) implies

$$
\sup _{\{x, \zeta\} \in U_{\eta, c}}\left|b_{+}\right| \leq e^{C_{a, b, c, \eta}\|\mathcal{V}\|_{L^{1}(a, b)}} \Rightarrow 1_{U_{\eta, c}} b_{+}=\mathcal{O}(1),
$$

and, taking into account the definition: $\chi_{+}=e^{i \zeta x} b_{+}$, it follows

$$
1_{U_{\eta, c}}(x, \zeta) \chi_{+}(x, \zeta)=e^{i \zeta x} \mathcal{O}(1) .
$$

Next, consider $\partial_{x} b_{+}=b_{+}^{\prime}$. For $x \in(c, b)$, it fulfills the equation

$$
b_{+}^{\prime}(x, \zeta)=-\int_{x}^{b} \partial_{x} \mathcal{K}_{+}(t, x, \zeta) \mathcal{V}(t) b_{+}(t, \zeta) d t, \quad x \in(c, b) .
$$

The regularity of the r.h.s. of (2.31) is a consequence of the properties of $b_{+}$and of the kernel $\partial_{x} \mathcal{K}_{+}$. In particular, making use of the above characterization of $b_{+}$, we get: $b_{+}^{\prime} \in \mathcal{C}_{x}^{0}\left((c, b), \mathcal{H}_{\zeta}\left(S_{\eta}\right)\right)$. Moreover, being $b_{+}$and $\partial_{x} \mathcal{K}_{+}$uniformly bounded for $\{x, \zeta\} \in U_{\eta, c}$ and $t \in(x, b)$, the r.h.s. of (2.31) is $\mathcal{O}(1)$. Then, a direct computation shows that

$$
1_{U_{\eta, c}}(x, \zeta) \partial_{x} \chi_{+}(x, \zeta)=e^{i \zeta x} \mathcal{O}(1+|\zeta|) .
$$
form

To discuss the case $x<c$, we notice that, when $x \in(-\infty, a)$, the solution $b_{+}$explicitly writes in the

$$
b_{+}(x, \zeta)=B_{+}(\zeta)+B_{-}(\zeta) e^{-2 i \zeta x} .
$$

The condition $b_{+} \in \mathcal{C}_{x}^{1}\left((c, b), \mathcal{H}_{\zeta}\left(S_{\eta}\right)\right)$ compels the coefficients $B_{ \pm}$to be holomorphic in $S_{\eta}$ with the only possible exception of the point $\zeta=0$; this leads: $b_{+} \in \mathcal{C}_{x}^{1}\left((-\infty, a), \mathcal{H}_{\zeta}\left(S_{\eta} \backslash\{0\}\right)\right)$. In $\zeta=0$, the maps $\zeta \rightarrow B_{ \pm}$may diverge, but, in such a case, a compensation between the different contributions at the r.h.s of (2.33) take place to assure the regularity of $\zeta \rightarrow 1_{U_{\eta, c}} b_{+}$in the origin. Therefore, $B_{ \pm}$may have, at most, a simple pole in $\zeta=0$ and the conditions

$$
\lim _{\zeta \rightarrow 0}\left(B_{+}(\zeta)+B_{-}(\zeta) e^{-2 i \zeta x}\right)=c_{0}, \quad \lim _{\zeta \rightarrow 0}-2 i \zeta B_{-}(\zeta) e^{-2 i \zeta x}=c_{1},
$$

holds for any $x \in(c, a)$. Since these are independent of $x$, the function $b_{+}$can be extended to: $b_{+} \in$ $\mathcal{C}_{x}^{1}\left((-\infty, a), \mathcal{H}_{\zeta}\left(S_{\eta}\right)\right)$

To conclude the proof, we need to extend the relations (2.30), (2.32) to the case of $x \in(-\infty, a)$ and $\zeta \in \overline{\mathbb{C}^{+}}$. According to (2.33) and (2.34), for any fixed $\zeta \in \overline{\mathbb{C}^{+}}$, the functions $b_{+}$and $b_{+}^{\prime}$ are uniformly bounded w.r.t. $x \in(-\infty, a)$. In particular, in a neighbourhood $\mathcal{B}_{1}(0) \cap \overline{\mathbb{C}^{+}}$of $\zeta=0$ we have

$$
1_{(-\infty, a)}(x) 1_{\mathcal{B}_{1}(0) \cap \overline{\mathbb{C}^{+}}}(\zeta) \partial_{x}^{i} b_{+}(x, \zeta)=\mathcal{O}(1), \quad i=0,1 .
$$

To obtain estimates as $|\zeta| \rightarrow \infty$, the high energy asymptotics of the coefficients $B_{ \pm}$is needed. The $x$-derivative of (2.33) is

$$
b_{+}^{\prime}(x, \zeta)=-2 i \zeta B_{-}(\zeta) e^{-2 i \zeta x} .
$$

As it has been previously shown, for $\{x, \zeta\} \in U_{\eta, c}$ it results $b_{+}^{\prime}(x, \zeta)=\mathcal{O}(1)$. Taking $x \in(c, a)$, and using (2.36), this implies: $\zeta B_{-}(\zeta)=\mathcal{O}(1)$ in the sense of the metric space $S_{\eta}$; it follows

$$
\zeta B_{-}(\zeta)=\mathcal{O}(1) \quad \text { in } \zeta \in \overline{\mathbb{C}^{+} \backslash \mathcal{B}_{1}(0) .}
$$

Similarly, since $b_{+}(x, \zeta)=\mathcal{O}(1)$ for $\{x, \zeta\} \in U_{\eta, c}$, taking $x \in(c, a)$ and using the relations (2.33) and (2.37), we get

$$
B_{+}(\zeta)=\mathcal{O}(1) \quad \text { in } \zeta \in \overline{\mathbb{C}^{+} \backslash \mathcal{B}_{1}(0)} .
$$


From these relations and the representations (2.33) and (2.36), we obtain

$$
1_{(-\infty, a)}(x) 1_{\overline{\mathbb{C}^{+}} \backslash \mathcal{B}_{1}(0)}(\zeta) \partial_{x}^{i} b_{+}(x, \zeta)=\mathcal{O}(1), \quad i=1,2
$$

Then, taking into account the definition $\chi_{+}=e^{i \zeta x} b_{+}$, the relations (2.20) follows from (2.30), (2.32), (2.39) and (2.39).

The Jost function, denoted in the following with $w(\zeta)$, is defined as the Wronskian associated with the couple $\left\{\chi_{+}(\cdot, \zeta), \chi_{-}(\cdot, \zeta)\right\}$. Setting

$$
w(f, g)=f g^{\prime}-f^{\prime} g
$$

we have

$$
w(\zeta)=\chi_{+}(\cdot, \zeta) \partial_{1} \chi_{-}(\cdot, \zeta)-\partial_{1} \chi_{+}(\cdot, \zeta) \chi_{-}(\cdot, \zeta)
$$

According to the definition of $\chi_{ \pm}$, this function is independent of the space variable, while due to the result of Proposition 2.1, $w(\zeta)$ is holomorphic w.r.t. $\zeta$ in an open half complex plane including $\overline{\mathbb{C}^{+}}$. The point spectrum of $Q_{0,0}(\mathcal{V})$ is defined by the solutions $z=\zeta^{2}$ to the problem: $w(\zeta)=0, \zeta \in \mathbb{C}^{+}$(e.g. in 31, Chp.5). Since $Q_{0,0}(\mathcal{V})$ is a selfadjoint Schrödinger operator with a short range potential, the point spectrum is non-degenerate and located on the negative real axis, while: $\sigma_{a c}\left(Q_{0,0}(\mathcal{V})\right)=[0,+\infty)$. Then, $w(\zeta)$ does not annihilates almost everywhere in the closed upper complex plane, with the only possible exceptions of a discrete subset of the positive imaginary axis. Next, consider $\zeta=k \in \mathbb{R}$ and let $w_{0}(k)$ be the Wronskian associated with $\left\{\chi_{+}(\cdot,-k), \chi_{-}(\cdot, k)\right\}$; the behavior of $w(k)$ on the real axis follows by using the relations

$$
\begin{aligned}
& \chi_{+}(\cdot, k)=\frac{1}{2 i k}\left(w_{0}^{*}(k) \chi_{-}(\cdot, k)-w(k) \chi_{-}(\cdot,-k)\right), \\
& \chi_{-}(\cdot, k)=\frac{1}{2 i k}\left(w_{0}(k) \chi_{+}(\cdot, k)-w(k) \chi_{+}(\cdot,-k)\right),
\end{aligned}
$$

expressing the Jost's solutions $\chi_{ \pm}(\cdot, k)$ in terms of the linearly independent couples $\chi_{-}(\cdot, \pm k)$ and $\chi_{+}(\cdot, \pm k)$ respectively (e.g. in [31, chp. 5). Plugging (2.43) into (2.42), indeed, it follows: $|w(k)|^{2}=$ $4 k^{2}+\left|w_{0}(k)\right|^{2}$, which entails

$$
|w(k)|^{2} \geq 4 k^{2}
$$

Let introduce the functions $\mathcal{G}^{z}(x, y)$ and $\mathcal{H}^{z}(x, y)$

$$
\begin{gathered}
\mathcal{G}^{z}(x, y)=\frac{1}{w(\zeta)}\left\{\begin{array}{ll}
\chi_{+}(x, \zeta) \chi_{-}(y, \zeta), & x \geq y, \\
\chi_{-}(x, \zeta) \chi_{+}(y, \zeta), & x<y,
\end{array} \quad z=\zeta^{2},\right. \\
\mathcal{H}^{z}(x, y)=-\frac{1}{w(\zeta)}\left\{\begin{array}{ll}
\chi_{+}(x, \zeta) \partial_{1} \chi_{-}(y, \zeta), & x \geq y, \\
\chi_{-}(x, \zeta) \partial_{1} \chi_{+}(y, \zeta), & x<y,
\end{array} \quad z=\zeta^{2},\right.
\end{gathered}
$$

Assume $\zeta \in \mathbb{C}^{+}$to be such that $w(\zeta) \neq 0$ and $y \in \mathbb{R}$; from the equation (2.18) and the relations (2.20), it follows that the maps $x \rightarrow \mathcal{G}^{z}(\cdot, y)$ and $x \rightarrow \mathcal{H}^{z}(\cdot, y)$ are exponentially decreasing as $|x-y| \rightarrow \infty$ (with a decreasing rate depending on $\operatorname{Im} \zeta$ ) and fulfill the boundary condition problems

$$
\begin{cases}\left(-\partial_{x}^{2}+\mathcal{V}-\zeta^{2}\right) \mathcal{G}^{z}(\cdot, y)=0 & \text { in } \mathbb{R} /\{y\} \\ \mathcal{G}^{z}\left(y^{+}, y\right)=\mathcal{G}^{z}\left(y^{-}, y\right), & \partial_{1} \mathcal{G}^{z}\left(y^{+}, y\right)-\partial_{1} \mathcal{G}^{z}\left(y^{-}, y\right)=-1\end{cases}
$$

and

$$
\begin{cases}\left(-\partial_{x}^{2}+\mathcal{V}-\zeta^{2}\right) \mathcal{H}^{z}(\cdot, y)=0 & \text { in } \mathbb{R} /\{y\} \\ \mathcal{H}^{z}\left(y^{+}, y\right)-\mathcal{H}^{z}\left(y^{-}, y\right)=1, & \partial_{1} \mathcal{H}^{z}\left(y^{+}, y\right)=\partial_{1} \mathcal{H}^{z}\left(y^{-}, y\right),\end{cases}
$$

For $z=\zeta^{2}$ s.t. $w(\zeta) \neq 0$ and $y \in\{a, b\}$, the functions $\mathcal{G}^{z}(\cdot, y), \mathcal{H}^{z}(\cdot, y)$ form a basis of the defect space $\mathcal{N}_{z}$, which writes as

$$
\mathcal{N}_{z}=\text { l.c. }\left\{\mathcal{G}^{z}(x, b), \mathcal{H}^{z}(x, b), \mathcal{G}^{z}(x, a), \mathcal{H}^{z}(x, a)\right\}
$$


According to the equation (2.47), $\mathcal{G}^{z}(\cdot, y)$ identifies with the integral kernel of $\left(Q_{0,0}(\mathcal{V})-z\right)^{-1}$, while, as a consequence of the definitions (2.45) $-(2.46)$ and the results of the Proposition 2.1 the maps $z \rightarrow \mathcal{G}^{z}(x, y)$, $z \rightarrow \mathcal{H}^{z}(x, y)$ are meromorphic in $\mathbb{C} \backslash \mathbb{R}_{+}$with a branch cut along the positive real axis and poles, corresponding to the points in $\sigma_{p}\left(Q_{0,0}(\mathcal{V})\right)$, located on the negative real axis. In particular, due to the inequality (2.44), these functions continuously extend up to the branch cut, both in the limits: $z \rightarrow k^{2} \pm i 0$, with the only possible exception of the point $z=0$.

In the case of defined positive potentials, it is possible to obtain uniform estimates of $\mathcal{G}^{z}(x, y)$ and $\mathcal{H}^{z}(x, y)$ up to the whole branch cut. Next, we assume $\mathcal{V}$ to fulfill the additional condition

$$
\langle u, \mathcal{V} u\rangle_{L^{2}(a, b)}>0 \quad \forall u \in L^{2}(\mathbb{R}),
$$

and introduce, for $\zeta \in \mathbb{C}^{+}$and $z=\zeta^{2}$, the functions

$$
G^{\zeta}(x, y)=\mathcal{G}^{\zeta^{2}}(x, y) ; \quad \partial_{1}^{i} H^{\zeta}(x, y)=\partial_{1}^{i} \mathcal{H}^{\zeta^{2}}(x, y)
$$

where the notation $\partial^{0} u=u$ is adopted. These are characterized as follows.

Lemma 2.3 Let $\mathcal{V}$ fulfill (1.9) and (2.50). For all $(x, y) \in \mathbb{R}^{2}, x \neq y$, the maps $\zeta \rightarrow G^{\zeta}(x, y)$ and $\zeta \rightarrow \partial_{1}^{i} H^{\zeta}(x, y), i=0,1$, defined according to 2.51) are holomorphic in $\mathbb{C}^{+}$and continuously extend to $\overline{\mathbb{C}^{+}}$. In particular, for $\zeta=k \in \mathbb{R}$, it results: $G^{k}(\cdot, y), H^{k}(\cdot, y) \in \mathcal{C}_{x}^{1}\left(\mathbb{R} \backslash\{y\}, \mathcal{C}_{k}^{0}(\mathbb{R})\right)$, while the relations

$$
G^{\zeta}(x, y)=e^{i \zeta|x-y|} \mathcal{O}\left(\frac{1}{1+|\zeta|}\right), \quad H^{\zeta}(x, y)=e^{i \zeta|x-y|} \mathcal{O}(1), \quad \partial_{1} H^{\zeta}(x, y)=e^{i \zeta|x-y|} \mathcal{O}(1+|\zeta|) .
$$

hold with $\mathcal{O}(\cdot)$ referred to the metric space $\mathbb{R}^{2} \times \overline{\mathbb{C}^{+}}$.

Proof. The conditions (1.9), (2.50) and the relation (2.44) prevent $w(\zeta)$ to have zeroes in $\overline{\mathbb{C}^{+} \backslash\{0\}}$. Computing $w(\zeta)$, we have

$$
w(\zeta)=\chi_{+}(a, \zeta) \partial_{1} \chi_{-}(a, \zeta)-\partial_{1} \chi_{+}(a, \zeta) \chi_{-}(a, \zeta)
$$

Using the exterior conditions (2.19), the coefficients $\partial_{1}^{j} \chi_{-}(a, \zeta), j=0,1$, are explicitly given by

$$
\chi_{-}(a, \zeta)=e^{-i \zeta a}, \quad \partial_{1} \chi_{-}(a, \zeta)=-i \zeta e^{-i \zeta a},
$$

For $x<b$, the function $\chi_{+}(\cdot, \zeta)$ writes as $\chi_{+}(x, \zeta)=e^{i \zeta x} b_{+}(x, \zeta)$, where $b_{+}(\cdot, \zeta)$ solves the equation (2.24) and can be represented as the sum of the Picard series: $b_{+}(\cdot, \zeta)=\sum_{n=0}^{+\infty} b_{+, n}(\cdot, \zeta)$ whose terms are defined by a recurrence relation given in (2.25). Under the condition (1.9), it has been shown that this series uniformly converges to $b_{+} \in \mathcal{C}_{x}^{1}\left((c, b), \mathcal{H}_{\zeta}\left(S_{\eta}\right)\right)$, being $(c, b)$ any interval including the point $a$ (see the proof of the Proposition 2.1); in particular the relations: $\partial_{1}^{j} b_{+}(\cdot, \zeta)=\mathcal{O}(1), j=0,1$, hold with the symbols $\mathcal{O}(\cdot)$ referring to the metric space: $(c, b) \times S_{\eta}$. Let $\zeta=0$; the relations (2.25) write as

$$
b_{+, n}(x, 0)=\int_{x}^{b}(t-x) \mathcal{V}(t) b_{+, n-1}(t, 0) d t, \quad n \in \mathbb{N}^{*}, x<b .
$$

Using the conditions: $\langle u, \mathcal{V} u\rangle_{L^{2}(a, b)}>0$ and $b_{+, 0}=1$, an induction argument leads to: $b_{+, n}(x, 0) \geq 0$ and $b_{+}(x, 0) \geq 1$. Taking the limit of (2.31) as $\zeta \rightarrow 0$, we get

$$
\partial_{x} b_{+}(x, 0)=-\int_{x}^{b} \mathcal{V}(t) b_{+}(t, 0) d t, \quad x<b .
$$

Since $b_{+}>0$ and $\mathcal{V}>0$, at least in a subset of $(a, b)$, we have: $\partial_{1} b_{+}(a, 0)<0$. With the notation introduced above, the equation (2.53) rephrases as

$$
w(\zeta)=-\partial_{1} b_{+}(a, \zeta)-2 i \zeta b_{+}(a, \zeta)
$$

Then, according to the conditions: $\partial_{1} b_{+}(a, 0) \neq 0$, and $b_{+}(\cdot, \zeta)=\mathcal{O}(1)$, we have: $w(\zeta)=-\partial_{1} b_{+}(a, \zeta)+$ $\mathcal{O}(\zeta)$ which implies $w(0) \neq 0$. 
As a consequence, the function $p(\zeta)$ defined by

$$
p(\zeta)=\frac{1+\zeta}{w(\zeta)}
$$

is bounded in any bounded set $\mathcal{B}_{R}(0) \cap \overline{\mathbb{C}^{+}}, R>0$. Moreover, using the equation (2.24), we have

$$
b_{+}(a, \zeta)=1+\int_{a}^{b} \frac{e^{2 i \zeta(t-x)}-1}{\zeta} \mathcal{V}(t) b_{+}(t, \zeta) d t
$$

since $b_{+}(\cdot, \zeta)$ is uniformly bounded for $\zeta \in \overline{\mathbb{C}^{+}}$, and $|1 / \zeta|\left|e^{2 i \zeta(t-x)}-1\right| \leq 2 /|\zeta|$, it follows: $\lim _{\zeta \rightarrow \infty, \zeta \in \overline{\mathbb{C}^{+}} b_{+}}(a, \zeta)=1$. Set $M=\sup _{\zeta \in \overline{\mathbb{C}^{+}}}\left|\partial_{1} b_{+}(a, \zeta)\right|$, and let $\tilde{R}>0$ be such that $|\zeta|\left|b_{+}(a, \zeta)\right| / M>$ 1 for any $\zeta \in \overline{\mathbb{C}^{+}} \backslash \mathcal{B}_{\tilde{R}}(0)$. From the representation (2.57) it follows

$$
\sup _{\zeta \in \mathbb{C}^{+} \backslash \mathcal{B}_{\tilde{R}}(0)}|p(\zeta)| \leq \frac{1}{M} \frac{1+|\zeta|}{2|\zeta| \frac{\left|b_{+}(a, \zeta)\right|}{M}-1} \lesssim 1 .
$$

Then, $p(\zeta)$ results uniformly bounded as $\zeta \in \overline{\mathbb{C}^{+}}$and we can write

$$
(w(\zeta))^{-1}=\mathcal{O}\left(\frac{1}{1+|\zeta|}\right)
$$

in the sense of the metric space $\overline{\mathbb{C}^{+}}$(see Definition 1.2$)$.

From the definitions (2.45)-(2.46), (2.51) and the result of Proposition2.1, the functions $\zeta \rightarrow G^{\zeta}(x, y)$ and $\zeta \rightarrow \partial_{1}^{i} H^{\zeta}(x, y), i=0,1$, are meromorphic in $\mathbb{C}^{+}$, while, the previous result implies that, in our assumptions, these maps have no poles in $\mathbb{C}^{+}$and continuously extend to the whole real axis. The relations (2.52) follows from (2.20) by using (2.61).

\subsection{Resolvent analysis.}

The results of the previous Sections and, in particular, the Krein's-like formula given in (2.16), allow a detailed resolvent analysis for the operators $Q_{\theta_{1}, \theta_{2}}(\mathcal{V})$. At this concern, we recall that the maps $z \rightarrow q(z, \mathcal{V})$ and $z \rightarrow \gamma\left(e_{i}, z, \mathcal{V}\right)$, appearing at the r.h.s. of (2.16), are holomorphic in $\mathbb{C} \backslash \sigma\left(Q_{0,0}(\mathcal{V})\right)$, while, from the definitions (2.13)-(2.13), the matrix coefficients in $A_{\theta_{1}, \theta_{2}}$ and $B_{\theta_{1}, \theta_{2}}$ are holomorphic functions of the parameters $\left(\theta_{1}, \theta_{2}\right)$ in the whole $\mathbb{C}^{2}$. Then

$$
d\left(z, \theta_{1}, \theta_{2}\right)=\operatorname{det}\left(B_{\theta_{1}, \theta_{2}} q(z, \mathcal{V})-A_{\theta_{1}, \theta_{2}}\right),
$$

defines an holomorphic function of the variables $\left(z, \theta_{1}, \theta_{2}\right)$ in $\mathbb{C} \backslash \sigma\left(Q_{0,0}(\mathcal{V})\right) \times \mathbb{C}^{2}$. Moreover, for any couple $\left(\theta_{1}, \theta_{2}\right)$, the set of singular points

$$
\mathcal{S}_{\theta_{1}, \theta_{2}}=\left\{z \in \mathbb{C} \mid d\left(z, \theta_{1}, \theta_{2}\right)=0\right\}
$$

is discrete. As a consequence, the representation (2.16) makes sense in the dense open set

$\mathbb{C} \backslash\left(\sigma\left(Q_{0,0}(\mathcal{V})\right) \cup \mathcal{S}_{\theta_{1}, \theta_{2}}\right)$. Let us fix $z \in \mathbb{C} \backslash\left(\sigma\left(Q_{0,0}(\mathcal{V})\right) \cup \mathcal{S}_{\tilde{\theta}_{1}, \tilde{\theta}_{2}}\right)$, for a given couple $\left(\tilde{\theta}_{1}, \tilde{\theta}_{2}\right) \in \mathbb{C}^{2} ;$ using the expansion

$$
d\left(z, \theta_{1}, \theta_{2}\right)=d\left(z, \tilde{\theta}_{1}, \tilde{\theta}_{2}\right)+\mathcal{O}\left(\theta_{1}-\tilde{\theta}_{1}\right)+\mathcal{O}\left(\theta_{2}-\tilde{\theta}_{2}\right)
$$

it results: $d\left(z, \theta_{1}, \theta_{2}\right) \neq 0$ for all $\left(\theta_{1}, \theta_{2}\right)$ in a suitable neighbourhood of $\left(\tilde{\theta}_{1}, \tilde{\theta}_{2}\right)$. This implies that, for any couple of parameters $\left(\tilde{\theta}_{1}, \tilde{\theta}_{2}\right)$, there exists $z \in \rho\left(Q_{\tilde{\theta}_{1}, \tilde{\theta}_{2}}(\mathcal{V})\right)$ and a positive constant $\delta$, possibly depending on $\left(\tilde{\theta}_{1}, \tilde{\theta}_{2}\right)$, such that: $z \in \rho\left(Q_{\theta_{1}, \theta_{2}}(\mathcal{V})\right)$ for all $\left(\theta_{1}, \theta_{2}\right) \in \mathcal{B}_{\delta}\left(\left(\tilde{\theta}_{1}, \tilde{\theta}_{2}\right)\right)$. Next, for such a $z$, consider the map $\left(\theta_{1}, \theta_{2}\right) \rightarrow\left(Q_{\theta_{1}, \theta_{2}}(\mathcal{V})-z\right)^{-1}$ defined for $\left(\theta_{1}, \theta_{2}\right) \in \mathcal{B}_{\delta}\left(\left(\tilde{\theta}_{1}, \tilde{\theta}_{2}\right)\right)$. Since $z \notin$ $\mathcal{S}_{\theta_{1}, \theta_{2}}$, the coefficients of the finite rank part at the r.h.s. of (2.16) are holomorphic w.r.t. $\left(\theta_{1}, \theta_{2}\right)$ and $\left(Q_{\theta_{1}, \theta_{2}}(\mathcal{V})-z\right)^{-1}$ forms an analytic family in $\mathcal{L}\left(L^{2}(\mathbb{R})\right)$. Then, $Q_{\theta_{1}, \theta_{2}}(\mathcal{V})$ is analytic in the sense of Kato, w.r.t. the parameters $\left(\theta_{1}, \theta_{2}\right)$. 
As this result suggests, when $\left(\theta_{1}, \theta_{2}\right)$ is close to the origin of $\mathbb{C}^{2}$, a part of the point spectrum $\sigma_{p}\left(Q_{\theta_{1}, \theta_{2}}(\mathcal{V})\right)$ is formed by non-degenerate eigenvalues holomorphically dependent on $\left(\theta_{1}, \theta_{2}\right)$ and converging, in the limit $\left(\theta_{1}, \theta_{2}\right) \rightarrow(0,0)$, to the corresponding points of $\sigma_{p}\left(Q_{0,0}(\mathcal{V})\right)$ (see the point $(i i)$ in the next Proposition 2.5). As an aside we notice that, for generic compactly supported potentials, new spectral points (not converging to $\sigma_{p}\left(Q_{0,0}(\mathcal{V})\right)$ ) may eventually arise in a complex neighbourhood of the origin, due to the interface conditions. Nevertheless, if the additional assumption of positive potentials (2.50) is adopted, it is possible to prove the identity $\sigma\left(Q_{\theta_{1}, \theta_{2}}(\mathcal{V})\right)=\sigma\left(Q_{0,0}(\mathcal{V})\right)$ provided that $\theta_{i=1,2}$ are small enough. To fix this point, we need appropriate estimates for the coefficients of the finite rank part in (2.16).

The relations (2.16) and (2.17) can be made explicit by computing the matrix representation of $\left(B_{\theta_{1}, \theta_{2}} q(z, \mathcal{V})-A_{\theta_{1}, \theta_{2}}\right)$ w.r.t. the basis $\left\{e_{j}\right\}_{j=1}^{4}$ and (2.49). Making use of the definition (2.7), a direct computation yields

$$
\gamma(\cdot, z, \mathcal{V})=\left(\begin{array}{llll}
1 & & & \\
& -1 & & \\
& & 1 & \\
& & & -1
\end{array}\right), \quad \text { with: } \begin{cases}\gamma\left(e_{1}, z, \mathcal{V}\right)=\mathcal{G}^{z}(x, b) ; & \gamma\left(e_{2}, z, \mathcal{V}\right)=-\mathcal{H}^{z}(x, b) \\
\gamma\left(e_{3}, z, \mathcal{V}\right)=\mathcal{G}^{z}(x, a) ; & \gamma\left(e_{4}, z, \mathcal{V}\right)=-\mathcal{H}^{z}(x, a)\end{cases}
$$

The matrix coefficients of $q(z, \mathcal{V})$ are related to the boundary values of the functions $\gamma\left(e_{i}, z, \mathcal{V}\right), i=1 \ldots 4$, as $x \rightarrow b^{ \pm}$or $x \rightarrow a^{ \pm}$. Using the definitions (2.45) and (2.46), it follows: $\partial_{1} \mathcal{G}^{z}(x, y)=\mathcal{H}^{z}(y, x)$; in particular, the boundary values at $x \rightarrow y^{ \pm}$are related by

$$
\partial_{1} \mathcal{G}^{z}\left(y^{ \pm}, y\right)=\mathcal{H}^{z}\left(y^{\mp}, y\right) \text {. }
$$

Using these relations and the boundary conditions in (2.47)-(2.48), a direct computation yields

$$
q(z, \mathcal{V})=\left(\begin{array}{cccc}
\mathcal{G}^{z}(b, b) & \frac{1}{2}-\mathcal{H}^{z}\left(b^{+}, b\right) & \mathcal{G}^{z}(b, a) & -\mathcal{H}^{z}(b, a) \\
\mathcal{H}^{z}\left(b^{+}, b\right)-\frac{1}{2} & -\partial_{1} \mathcal{H}^{z}(b, b) & \mathcal{H}^{z}(a, b) & -\partial_{1} \mathcal{H}^{z}(b, a) \\
\mathcal{G}^{z}(a, b) & -\mathcal{H}^{z}(a, b) & \mathcal{G}^{z}(a, a) & -\left(\frac{1}{2}+\mathcal{H}^{z}\left(a^{-}, a\right)\right) \\
\mathcal{H}^{z}(b, a) & -\partial_{1} \mathcal{H}^{z}(a, b) & \mathcal{H}^{z}\left(a^{-}, a\right)+\frac{1}{2} & -\partial_{1} \mathcal{H}^{z}(a, a)
\end{array}\right) .
$$

Lemma 2.4 Let the matrix $\left(B_{\theta_{1}, \theta_{2}} q(z, \mathcal{V})-A_{\theta_{1}, \theta_{2}}\right)$ be defined according to the relations (2.67), (2.12)(2.13) and assume $\mathcal{V}$ to fulfill the conditions (1.9), (2.50). There exists $\delta>0$ such that, for all $\left(\theta_{1}, \theta_{2}\right) \in$ $\mathcal{B}_{\delta}((0,0)),\left(B_{\theta_{1}, \theta_{2}} q(z, \mathcal{V})-A_{\theta_{1}, \theta_{2}}\right)$ is invertible in $z \in \mathbb{C} / \mathbb{R}_{+}$. The coefficients of the inverse matrix are holomorphic w.r.t. $\left(z, \theta_{1}, \theta_{2}\right)$ with: $\left(\theta_{1}, \theta_{2}\right) \in \mathcal{B}_{\delta}((0,0)), z \in \mathbb{C} / \mathbb{R}_{+} ;$they have continuous extensions to the branch cut both in the limits $z=k^{2}+i \varepsilon, \varepsilon \rightarrow 0^{ \pm}$.

Proof. Using the notation introduced in (2.51), for $z=\zeta^{2}, \zeta \in \mathbb{C}^{+}$, a direct computation leads to

$\left(B_{\theta_{1}, \theta_{2}} q(z, \mathcal{V})-A_{\theta_{1}, \theta_{2}}\right)=$

$$
\begin{gathered}
\left(\begin{array}{cccc}
\beta\left(\theta_{2}\right)\left(H^{\zeta}\left(b^{+}, b\right)-\frac{1}{2}\right) & -\beta\left(\theta_{2}\right) \partial_{1} H^{\zeta}(b, b) & \beta\left(\theta_{2}\right) H^{\zeta}(a, b) & -\beta\left(\theta_{2}\right) \partial_{1} H^{\zeta}(b, a) \\
\beta\left(\theta_{1}\right) G^{\zeta}(b, b) & \beta\left(\theta_{1}\right)\left(\frac{1}{2}-H^{\zeta}\left(b^{+}, b\right)\right) & \beta\left(\theta_{1}\right) G^{\zeta}(b, a) & -\beta\left(\theta_{1}\right) H^{\zeta}(b, a) \\
\beta\left(-\theta_{2}\right) H^{\zeta}(b, a) & -\beta\left(-\theta_{2}\right) \partial_{1} H^{\zeta}(a, b) & \beta\left(-\theta_{2}\right)\left(H^{\zeta}\left(a^{-}, a\right)+\frac{1}{2}\right) & -\beta\left(-\theta_{2}\right) \partial_{1} H^{\zeta}(a, a) \\
\beta\left(-\theta_{1}\right) G^{\zeta}(a, b) & -\beta\left(-\theta_{1}\right) H^{\zeta}(a, b) & \beta\left(-\theta_{1}\right) G^{\zeta}(a, a) & -\beta\left(-\theta_{1}\right)\left(\frac{1}{2}+H^{\zeta}\left(a^{-}, a\right)\right)
\end{array}\right) \\
-\left(\begin{array}{ccc}
\alpha\left(\theta_{2}\right) & & \\
& \alpha\left(\theta_{1}\right) & \\
& \alpha\left(-\theta_{2}\right) & \\
& & \alpha\left(-\theta_{1}\right)
\end{array}\right)
\end{gathered}
$$

where $\alpha(\theta)$ and $\beta(\theta)$ are defined by

$$
\alpha(\theta)=1+e^{\frac{\theta}{2}}, \quad \beta(\theta)=1-e^{\frac{\theta}{2}} .
$$

As consequence of the Lemma 2.3. for defined positive potentials the above relation rephrases as

$$
\begin{aligned}
& \left(B_{\theta_{1}, \theta_{2}} q(z, \mathcal{V})-A_{\theta_{1}, \theta_{2}}\right)= \\
& \left(\begin{array}{cccc}
\beta\left(\theta_{2}\right) \mathcal{O}(1)-\alpha\left(\theta_{2}\right) & \beta\left(\theta_{2}\right) \mathcal{O}(1+|\zeta|) & \beta\left(\theta_{2}\right) e^{i \zeta(b-a)} \mathcal{O}(1) & \beta\left(\theta_{2}\right) e^{i \zeta(b-a)} \mathcal{O}(1+|\zeta|) \\
\beta\left(\theta_{1}\right) \mathcal{O}\left(\frac{1}{1+|\zeta|}\right) & \beta\left(\theta_{1}\right) \mathcal{O}(1)-\alpha\left(\theta_{1}\right) & \beta\left(\theta_{1}\right) e^{i \zeta(b-a)} \mathcal{O}\left(\frac{1}{1+|\zeta|}\right) & \beta\left(\theta_{1}\right) e^{i \zeta(b-a)} \mathcal{O}(1) \\
\beta\left(-\theta_{2}\right) e^{i \zeta(b-a)} \mathcal{O}(1) & \beta\left(-\theta_{2}\right) e^{i \zeta(b-a)} \mathcal{O}(1+|\zeta|) & \beta\left(-\theta_{2}\right) \mathcal{O}(1)-\alpha\left(-\theta_{2}\right) & \beta\left(-\theta_{2}\right) \mathcal{O}(1+|\zeta|) \\
\beta\left(-\theta_{1}\right) e^{i \zeta(b-a)} \mathcal{O}\left(\frac{1}{1+|\zeta|}\right) & \beta\left(-\theta_{1}\right) e^{i \zeta(b-a)} \mathcal{O}(1) & \beta\left(-\theta_{1}\right) \mathcal{O}\left(\frac{1}{1+|\zeta|}\right) & \beta\left(-\theta_{1}\right) \mathcal{O}(1)-\alpha\left(-\theta_{1}\right)
\end{array}\right)
\end{aligned}
$$


being the symbols $\mathcal{O}(\cdot)$ referred to the metric space $\overline{\mathbb{C}^{+}}$and defining holomorphic functions of $\zeta \in$ $\mathbb{C}^{+}$with continuous extension the real axis. Due to the definition of $\alpha(\theta), \beta(\theta)$, the coefficients of $\left(B_{\theta_{1}, \theta_{2}} q(z, \mathcal{V})-A_{\theta_{1}, \theta_{2}}\right)$ result separately w.r.t. $\left(\theta_{1}, \theta_{2}\right) \in \mathbb{C}^{2}, z \in \mathbb{C} / \mathbb{R}_{+}$, and admit, for each couple $\left(\theta_{1}, \theta_{2}\right)$, continuous extensions to the branch cut. In particular, setting $\zeta=k \in \mathbb{R}_{ \pm}$at the r.h.s. of (2.70) corresponds to consider the limits of $\left(B_{\theta_{1}, \theta_{2}} q(z, \mathcal{V})-A_{\theta_{1}, \theta_{2}}\right)_{i j}$ for $z \rightarrow k^{2} \pm i 0$ respectively. Making use of this expression, and taking into account (2.69), a determinant's expansion follows

$$
d\left(z, \theta_{1}, \theta_{2}\right)=4\left(1+\cosh \frac{\theta_{1}}{2}\right)\left(1+\cosh \frac{\theta_{2}}{2}\right)+\mathcal{O}\left(\theta_{1}\right)+\mathcal{O}\left(\theta_{2}\right)
$$

where $\mathcal{O}\left(\theta_{i}\right)$, being referred to the metric space $\mathcal{B}_{1}((0,0)) \times \mathbb{C}$, defines holomorphic functions w.r.t. $\left(\theta_{1}, \theta_{2}\right) \in \mathcal{B}_{1}((0,0))$ and $z \in \mathbb{C} / \mathbb{R}_{+}$, allowing continuous extensions to the branch cut in the abovespecified sense. According to the Definition $1.2 \mathcal{O}\left(\theta_{i}\right)$ writes as

$$
\mathcal{O}\left(\theta_{i}\right)=\theta_{i} p\left(z, \theta_{1}, \theta_{2}\right)
$$

with $p\left(z, \theta_{1}, \theta_{2}\right)$ uniformly bounded in $\mathcal{B}_{1}((0,0)) \times \mathbb{C}$. Therefore $\delta>0$ exists such that, when $\left(\theta_{1}, \theta_{2}\right) \in$ $\mathcal{B}_{\delta}((0,0))$, it results $\left|d\left(z, \theta_{1}, \theta_{2}\right)\right|>1$ for all $z \in \mathbb{C} / \mathbb{R}_{+}$. In these conditions, the matrix $\left(B_{\theta_{1}, \theta_{2}} q(z, \mathcal{V})-A_{\theta_{1}, \theta_{2}}\right)$ is invertible and the coefficients $\left(B_{\theta_{1}, \theta_{2}} q(z, \mathcal{V})-A_{\theta_{1}, \theta_{2}}\right)_{i j}^{-1}$ are separately holomorphic w.r.t. $\left(\theta_{1}, \theta_{2}\right) \in$ $\mathcal{B}_{\delta}((0,0)), z \in \mathbb{C} / \mathbb{R}_{+}$, having continuous extensions to the whole branch cut, both in the limits $z=k^{2}+i \varepsilon$, $\varepsilon \rightarrow 0^{ \pm}$.

We are now in the position to develop the spectral analysis for the operators $Q_{\theta_{1}, \theta_{2}}(\mathcal{V})$ starting from the resolvent's formula (2.16).

Proposition 2.5 Let $Q_{\theta_{1}, \theta_{2}}(\mathcal{V})$ be defined according to (1.9), (1.11). The operator's spectrum characterizes as follows:

i) For any $\left(\theta_{1}, \theta_{2}\right) \in \mathbb{C}^{2}$, the essential part of the spectrum is $\sigma_{\text {ess }}\left(Q_{\theta_{1}, \theta_{2}}(\mathcal{V})\right)=\mathbb{R}_{+}$.

ii) Let $E_{0}$ be an eigenvalue of $Q_{0,0}(\mathcal{V})$ and assume $\varepsilon_{0}>0$ small enough; for any fixed $\varepsilon \in\left(0, \varepsilon_{0}\right)$ it exists $\delta_{\varepsilon}>0$ depending on $\varepsilon$ s.t.: for any $\left(\theta_{1}, \theta_{2}\right) \in \mathcal{B}_{\delta_{\varepsilon}}((0,0)) \cap \mathbb{C}^{2}$ it exists an unique nondegenerate and discrete eigenvalue $E\left(\theta_{1}, \theta_{2}\right) \in \sigma\left(Q_{\theta_{1}, \theta_{2}}(\mathcal{V})\right) \cap \mathcal{B}_{\varepsilon}\left(E_{0}\right)$. Moreover, the function $E\left(\theta_{1}, \theta_{2}\right)$ is holomorphic w.r.t. $\left(\theta_{1}, \theta_{2}\right)$ in $\mathcal{B}_{\delta_{\varepsilon}}((0,0))$.

If, in addition, $\mathcal{V}$ is assumed to be defined positive, fulfilling (2.50), then:

iii) It exists $\delta>0$ s.t., for all $\left(\theta_{1}, \theta_{2}\right) \in \mathcal{B}_{\delta}((0,0)), \sigma\left(Q_{\theta_{1}, \theta_{2}}(\mathcal{V})\right)$ is purely absolutely continuous and coincide with the positive real axis.

Proof. With the notation introduced above, let $z \in \mathbb{C} \backslash\left(\sigma\left(Q_{0,0}(\mathcal{V})\right) \cup \mathcal{S}_{\theta_{1}, \theta_{2}}\right)$; the representation (2.16) implies that the difference $\left(Q_{\theta_{1}, \theta_{2}}(\mathcal{V})-z\right)^{-1}-\left(Q_{0,0}(\mathcal{V})-z\right)^{-1}$ is a finite rank operator. Then, the first statement of the Proposition follows by adapting the Weyl's theorem to the non-selfadjoint framework (for this point, we refer to [27], Sec. XIII.4, Lemma 3 and the strong spectral mapping theorem).

In the unperturbed case, $Q_{0,0}(\mathcal{V})$ is a $1 \mathrm{D}$ Schrödinger operator with a short range potential. Its spectrum has a purely absolutely continuous part on the positive real axis, and possible non-degenerate eigenvalues located on the negative real axis, without accumulation points. Then, the second statement is a direct consequence of the Kato-Rellich theorem, since $Q_{\theta_{1}, \theta_{2}}(\mathcal{V})$ is Kato-analytic w.r.t. the parameters.

When $\mathcal{V}$ is a defined positive potential, $\sigma\left(Q_{0,0}(\mathcal{V})\right)=\sigma_{a c}\left(Q_{0,0}(\mathcal{V})\right)=\mathbb{R}_{+}$, while the point spectrum is empty. The spectrum $\sigma\left(Q_{\theta_{1}, \theta_{2}}(\mathcal{V})\right)$ corresponds to the subset of the complex plane where the map $z \rightarrow \mathcal{G}_{\theta_{1}, \theta_{2}}^{z}(x, y)$ (defined in eq. (2.17)) is not holomorphic. According to the result of the Lemma 2.3. the functions $\mathcal{G}^{z}(x, y)$ and $\mathcal{H}^{z}(x, y)$, appearing at the r.h.s. of (2.17) are $z$-holomorphic in $\mathbb{C} / \mathbb{R}_{+}$and continuously extend to the whole branch cut both in the limits $z=k^{2}+i \varepsilon, \varepsilon \rightarrow 0^{ \pm}$. As shown in Lemma 2.4, the same hold for the coefficients of $\left(B_{\theta_{1}, \theta_{2}} q(z, \mathcal{V})-A_{\theta_{1}, \theta_{2}}\right)^{-1}$, provided that $\left(\theta_{1}, \theta_{2}\right)$ is close enough to the origin in $\mathbb{C}^{2}$. In particular, these are $z$-holomorphic in $\mathbb{C} / \mathbb{R}_{+}$and have continuous extensions to the whole branch cut. Then, for $\mathcal{V}$ defined positive, the map $z \rightarrow \mathcal{G}_{\theta_{1}, \theta_{2}}^{z}(x, y)$ is holomorphic in $\mathbb{C} / \mathbb{R}_{+}$ and have continuous extensions as $z \rightarrow \mathbb{R}_{+}$, both in the limits $z=k^{2}+i \varepsilon, \varepsilon \rightarrow 0^{ \pm}$. This yields: $\sigma\left(Q_{\theta_{1}, \theta_{2}}(\mathcal{V})\right)=\sigma_{a c}\left(Q_{\theta_{1}, \theta_{2}}(\mathcal{V})\right)=\mathbb{R}_{+}$ 


\subsection{Generalized eigenfunctions expansion.}

Let $\psi_{-}\left(\cdot, k, \theta_{1}, \theta_{2}\right)$ denote the generalized eigenfunction of the operator $Q_{\theta_{1}, \theta_{2}}(\mathcal{V})$, describing an incoming wave function of momentum $k$; this is a solution to the boundary value problem

$$
\begin{cases}\left(-\partial_{x}^{2}+\mathcal{V}\right) u=k^{2} u, & \text { for } x \in \mathbb{R} \backslash\{a, b\}, k \in \mathbb{R}, \\ e^{-\frac{\theta_{1}}{2}} u\left(b^{+}, \zeta, \theta_{1}, \theta_{2}\right)=u\left(b^{-}, \zeta, \theta_{1}, \theta_{2}\right), & e^{-\frac{\theta_{2}}{2}} u^{\prime}\left(b^{+}, \zeta, \theta_{1}, \theta_{2}\right)=u^{\prime}\left(b^{-}, \zeta, \theta_{1}, \theta_{2}\right), \\ e^{-\frac{\theta_{1}}{2}} u\left(a^{-}, \zeta, \theta_{1}, \theta_{2}\right)=u\left(a^{+}, \zeta, \theta_{1}, \theta_{2}\right), & e^{-\frac{\theta_{2}}{2}} u^{\prime}\left(a^{-}, \zeta, \theta_{1}, \theta_{2}\right)=u\left(a^{+}, \zeta, \theta_{1}, \theta_{2}\right),\end{cases}
$$

fulfilling the exterior conditions

$$
\left.\psi_{-}\left(x, k, \theta_{1}, \theta_{2}\right)\right|_{\substack{x<a \\ k>0}}=e^{i k x}+R\left(k, \theta_{1}, \theta_{2}\right) e^{-i k x},\left.\quad \psi_{-}\left(x, k, \theta_{1}, \theta_{2}\right)\right|_{\substack{x>b \\ k>0}}=T\left(k, \theta_{1}, \theta_{2}\right) e^{i k x},
$$

and

$$
\left.\psi_{-}\left(x, k, \theta_{1}, \theta_{2}\right)\right|_{\substack{x<a \\ k<0}}=T\left(k, \theta_{1}, \theta_{2}\right) e^{i k x},\left.\quad \psi_{-}\left(x, k, \theta_{1}, \theta_{2}\right)\right|_{\substack{x>b \\ k<0}}=e^{i k x}+R\left(k, \theta_{1}, \theta_{2}\right) e^{-i k x},
$$

where $R$ and $T$ are the reflection and transmission coefficients. In the case $\left(\theta_{1}, \theta_{2}\right)=(0,0), \psi_{-}(\cdot, k, 0,0)$ is a generalized eigenfunction of the selfadjoint model $Q_{0,0}(\mathcal{V})$. In what follows we adopt the simplified notation: $\psi_{-}(\cdot, k, 0,0)=\psi_{-}(\cdot, k)$. These functions are expressed in terms of the corresponding Jost's solutions as

$$
\psi_{-}(x, k)= \begin{cases}-\frac{2 i k}{w(k)} \chi_{+}(x, k), & \text { for } k \geq 0, \\ \frac{2 i k}{w(-k)} \chi_{-}(x,-k), & \text { for } k<0,\end{cases}
$$

(e.g. in [31]). In the case of defined positive potentials, an approach similar to the one leading to the Krein-like resolvent formula (2.10) allows to obtain an expansion for the difference: $\psi_{-}\left(\cdot, k, \theta_{1}, \theta_{2}\right)-\psi_{-}(\cdot, k)$ for $\left(\theta_{1}, \theta_{2}\right) \rightarrow(0,0)$. To this aim, we need an explicit expression of the finite rank terms, appearing at the r.h.s. of (2.16), in the limits where $z$ approaches the branch cut. This can be done by using the results of Lemmas 2.3 and 2.4. Adopting the notation introduced in (2.51), let us define

$$
\left\{g\left(e_{i}, \zeta, \mathcal{V}\right)\right\}_{i=1}^{4}=\left\{G^{\zeta}(\cdot, b),-H^{\zeta}(\cdot, b), G^{\zeta}(\cdot, a),-H^{\zeta}(\cdot, a)\right\}
$$

we get, for $\zeta \in \mathbb{C}^{+}$and $z=\zeta^{2}$, the identity: $\gamma\left(e_{i}, z, \mathcal{V}\right)=g\left(e_{i}, \zeta, \mathcal{V}\right)$; due to Lemma 2.3. the limits of $g\left(e_{i}, \zeta, \mathcal{V}\right)$ as $\zeta \rightarrow k \in \mathbb{R}_{ \pm}$exist and corresponds to the limits of $\gamma\left(e_{i}, z, \mathcal{V}\right)$ as $z \rightarrow k^{2} \pm i 0$ respectively. Namely, we have

$$
\lim _{z \rightarrow k^{2} \pm i 0} \gamma\left(e_{i}, z, \mathcal{V}\right)=\left\{\begin{array}{l}
\left.g\left(e_{i}, k, \mathcal{V}\right)\right|_{k \in \mathbb{R}_{+}} \\
\left.g\left(e_{i}, k, \mathcal{V}\right)\right|_{k \in \mathbb{R}_{-}}
\end{array}\right.
$$

The coefficients $\left(B_{\theta_{1}, \theta_{2}} q(z, \mathcal{V})-A_{\theta_{1}, \theta_{2}}\right)_{i j}^{-1}$ have been considered in the Lemma 2.4 where their regularity w.r.t. the $z$ and the extensions to the branch cut have been investigated. To get further insights on the structure of the inverse matrix, we use the explicit form of $\left(B_{\theta_{1}, \theta_{2}} q(z, \mathcal{V})-A_{\theta_{1}, \theta_{2}}\right)$ given in (2.68) $-(2.70)$. In what follows, $\mathcal{M}\left(\zeta, \theta_{1}, \theta_{2}\right)$ denotes the r.h.s. of (2.68)

$$
\left(B_{\theta_{1}, \theta_{2}} q(z, \mathcal{V})-A_{\theta_{1}, \theta_{2}}\right)=\mathcal{M}\left(\zeta, \theta_{1}, \theta_{2}\right), \quad \zeta \in \mathbb{C}^{+}, z=\zeta^{2} .
$$

In the assumption (2.50), the matrix $\mathcal{M}\left(\zeta, \theta_{1}, \theta_{2}\right)$ continuously extends extends to $\zeta \in \overline{\mathbb{C}^{+}}$and taking its limits for $\zeta \rightarrow k \in \mathbb{R}_{ \pm}$corresponds to consider the limits of $\left(B_{\theta_{1}, \theta_{2}} q(z, \mathcal{V})-A_{\theta_{1}, \theta_{2}}\right)_{i j}$ as $z \rightarrow k^{2} \pm i 0$ respectively. This yields

$$
\lim _{z \rightarrow k^{2} \pm i 0}\left(B_{\theta_{1}, \theta_{2}} q(z, \mathcal{V})-A_{\theta_{1}, \theta_{2}}\right)=\left\{\begin{array}{l}
\left.\mathcal{M}\left(k, \theta_{1}, \theta_{2}\right)\right|_{k \in \mathbb{R}_{+}^{*}} \\
\left.\mathcal{M}\left(k, \theta_{1}, \theta_{2}\right)\right|_{k \in \mathbb{R}_{-}}
\end{array}\right.
$$


In particular, making use of (2.70), we have

$$
\begin{aligned}
\mathcal{M}\left(k, \theta_{1}, \theta_{2}\right)= & \\
& \left(\begin{array}{cccc}
\beta\left(\theta_{2}\right) \mathcal{O}(1)-\alpha\left(\theta_{2}\right) & \beta\left(\theta_{2}\right) \mathcal{O}(1+|k|) & \beta\left(\theta_{2}\right) \mathcal{O}(1) & \beta\left(\theta_{2}\right) \mathcal{O}(1+|k|) \\
\beta\left(\theta_{1}\right) \mathcal{O}\left(\frac{1}{1+|k|}\right) & \beta\left(\theta_{1}\right) \mathcal{O}(1)-\alpha\left(\theta_{1}\right) & \beta\left(\theta_{1}\right) \mathcal{O}\left(\frac{1}{1+|k|}\right) & \beta\left(\theta_{1}\right) \mathcal{O}(1) \\
\beta\left(-\theta_{2}\right) \mathcal{O}(1) & \beta\left(-\theta_{2}\right) \mathcal{O}(1+|k|) & \beta\left(-\theta_{2}\right) \mathcal{O}(1)-\alpha\left(-\theta_{2}\right) & \beta\left(-\theta_{2}\right) \mathcal{O}(1+|k|) \\
\beta\left(-\theta_{1}\right) \mathcal{O}\left(\frac{1}{1+|k|}\right) & \beta\left(-\theta_{1}\right) \mathcal{O}(1) & \beta\left(-\theta_{1}\right) \mathcal{O}\left(\frac{1}{1+\mid k}\right) & \beta\left(-\theta_{1}\right) \mathcal{O}(1)-\alpha\left(-\theta_{1}\right)
\end{array}\right) .
\end{aligned}
$$

From the Lemma 2.4 this matrix is invertible whenever $\left(\theta_{1}, \theta_{2}\right) \in \mathcal{B}_{\delta}((0,0))$ with $\delta$ small enough; under such a condition, indeed, the determinant's expansion

$$
\begin{aligned}
& \operatorname{det} \mathcal{M}\left(k, \theta_{1}, \theta_{2}\right)=\operatorname{det}\left(-A_{\theta_{1}, \theta_{2}}\right)+\mathcal{O}\left(\theta_{1}\right)+\mathcal{O}\left(\theta_{2}\right), \\
& \operatorname{det}\left(-A_{\theta_{1}, \theta_{2}}\right)=4\left(1+\cosh \frac{\theta_{1}}{2}\right)\left(1+\cosh \frac{\theta_{2}}{2}\right),
\end{aligned}
$$

(see the relation (2.71) $)$ implies: $\left|\operatorname{det} \mathcal{M}\left(k, \theta_{1}, \theta_{2}\right)\right|>1$. Then, a direct computation leads to

$$
\mathcal{M}^{-1}\left(k, \theta_{1}, \theta_{2}\right)=\frac{1}{\operatorname{det} \mathcal{M}\left(k, \theta_{1}, \theta_{2}\right)}\left[\operatorname{det}\left(A_{\theta_{1}, \theta_{2}}\right) \operatorname{diag}\left\{\lambda_{i}\right\}+M\left(k, \theta_{1}, \theta_{2}\right)\right],
$$

where diag $\left(\lambda_{i}\right)$, the main term in (2.82), is the $\mathbb{C}^{4,4}$ diagonal matrix defined by the coefficients

$$
\left\{\lambda_{i}\right\}_{i=1}^{4}=\left\{\frac{-1}{\alpha\left(\theta_{2}\right)}, \frac{-1}{\alpha\left(\theta_{1}\right)}, \frac{-1}{\alpha\left(-\theta_{2}\right)}, \frac{-1}{\alpha\left(-\theta_{1}\right)}\right\}
$$

while the remainder is

$$
M\left(k, \theta_{1}, \theta_{2}\right)=\left(\begin{array}{cccc}
\mathcal{O}\left(\theta_{1}\right)+\mathcal{O}\left(\theta_{2}\right) & \mathcal{O}\left(\theta_{2}(1+|k|)\right) & \mathcal{O}\left(\theta_{2}\right) & \mathcal{O}\left(\theta_{2}(1+|k|)\right) \\
\mathcal{O}\left(\frac{\theta_{1}}{1+|k|}\right) & \mathcal{O}\left(\theta_{1}\right)+\mathcal{O}\left(\theta_{2}\right) & \mathcal{O}\left(\frac{\theta_{1}}{1+|k|}\right) & \mathcal{O}\left(\theta_{1}\right) \\
\mathcal{O}\left(\theta_{2}\right) & \mathcal{O}\left(\theta_{2}(1+|k|)\right) & \mathcal{O}\left(\theta_{1}\right)+\mathcal{O}\left(\theta_{2}\right) & \mathcal{O}\left(\theta_{2}(1+|k|)\right) \\
\mathcal{O}\left(\frac{\theta_{1}}{1+|k|}\right) & \mathcal{O}\left(\theta_{1}\right) & \mathcal{O}\left(\frac{\theta_{1}}{1+|k|}\right) & \mathcal{O}\left(\theta_{1}\right)+\mathcal{O}\left(\theta_{2}\right)
\end{array}\right)
$$

Here, the symbols $\mathcal{O}(\cdot)$ are referred to the metric space $\mathcal{B}_{\delta}((0,0)) \times \mathbb{R}$ and, being obtained from the calculus of the inverse matrix $\left(B_{\theta_{1}, \theta_{2}} q(z, \mathcal{V})-A_{\theta_{1}, \theta_{2}}\right)^{-1}$, denotes polynomial expressions depending on the functions: $\alpha\left( \pm \theta_{i}\right), \beta\left( \pm \theta_{i}\right), G^{\zeta}(x, y), \partial_{1}^{i} H^{\zeta}(x, y)$, with $x, y \in\{a, b\}$ and $i=0,1$. Then, as a consequence of Lemma 2.3, these terms are holomorphic w.r.t. the parameters $\left(\theta_{1}, \theta_{2}\right) \in \mathcal{B}_{\delta}((0,0))$ and continuous w.r.t. $k \in \mathbb{R}$.

Proposition 2.6 Assume $\left(\theta_{1}, \theta_{2}\right) \in \mathcal{B}_{\delta}((0,0))$ with $\delta>0$ small enough, and let $\mathcal{V}$ be defined according to (1.9), (2.50). The solutions $\psi_{-}\left(\cdot, k, \theta_{1}, \theta_{2}\right)$ to the generalized eigenfunctions problem 2.73), 2.74)2.75) allow the representation

$\psi_{-}\left(\cdot, k, \theta_{1}, \theta_{2}\right)= \begin{cases}\psi_{-}(\cdot, k)-\sum_{i, j=1}^{4}\left[\mathcal{M}^{-1}\left(k, \theta_{1}, \theta_{2}\right) B_{\theta_{1}, \theta_{2}}\right]_{i j}\left[\Gamma_{1} \psi_{-}(\cdot, k)\right]_{j} g\left(e_{i}, k, \mathcal{V}\right), & \text { for } k \geq 0, \\ \psi_{-}(\cdot, k)-\sum_{i, j=1}^{4}\left[\mathcal{M}^{-1}\left(-k, \theta_{1}, \theta_{2}\right) B_{\theta_{1}, \theta_{2}}\right]_{i j}\left[\Gamma_{1} \psi_{-}(\cdot, k)\right]_{j} g\left(e_{i},-k, \mathcal{V}\right), & \text { for } k<0 .\end{cases}$

The functions $\psi_{-}\left(x, k, \theta_{1}, \theta_{2}\right)$ are $\mathcal{C}^{1}$-continuous w.r.t. $x \in \mathbb{R} /\{a, b\}, k$-continuous in $\mathbb{R}$ and holomorphic w.r.t. the parameters $\left(\theta_{1}, \theta_{2}\right)$ in $\mathcal{B}_{\delta}((0,0))$.

Proof. We start considering the case $k \geq 0$. According to the definition of $\psi_{-}(\cdot, k)$ and $g\left(e_{i}, k, \mathcal{V}\right)$, the function at the r.h.s. of (2.85) solves the equation

$$
\left(-\partial_{x}^{2}+\mathcal{V}\right) u=k^{2} u, \quad \text { for } x \in \mathbb{R} \backslash\{a, b\}, k \in \mathbb{R},
$$

and fulfills the conditions (2.74) and (2.75). Set: $\psi_{-}\left(\cdot, k, \theta_{1}, \theta_{2}\right)=\phi-\psi$ with

$$
\begin{aligned}
& \phi=\psi_{-}(\cdot, k) \\
& \psi=\sum_{i, j=1}^{4}\left[\mathcal{M}^{-1}\left(k, \theta_{1}, \theta_{2}\right) B_{\theta_{1}, \theta_{2}}\right]_{i j}\left[\Gamma_{1} \phi\right]_{j} g\left(e_{i}, k, \mathcal{V}\right) .
\end{aligned}
$$


The function $\psi$ can be pointwise approximated by elements of the defect spaces $\mathcal{N}_{z}$ as $z \rightarrow k^{2}+i 0$. With the notation introduced in (2.79) and (2.77), let $\psi_{z}$ be defined by

$$
\psi_{z}=\sum_{i, j=1}^{4}\left[\mathcal{M}^{-1}\left(\zeta, \theta_{1}, \theta_{2}\right) B_{\theta_{1}, \theta_{2}}\right]_{i j}\left[\Gamma_{1} \phi\right]_{j} g\left(e_{i}, \zeta, \mathcal{V}\right), \quad \zeta \in \mathbb{C}^{+}, z=\zeta^{2}
$$

it results $\psi_{z} \in \mathcal{N}_{z}$ and $\lim _{z \rightarrow k^{2}+i 0} \psi_{z}=\psi$. Since $\psi_{-}(\cdot, k)$ is $\mathcal{C}_{x}^{1}$-continuous in $\mathbb{R}$, we have: $\Gamma_{0} \phi=0$ and the following relation holds

$$
\begin{aligned}
\mathcal{M}\left(k, \theta_{1}, \theta_{2}\right) \Gamma_{0}(\phi-\psi) & =-\mathcal{M}\left(k, \theta_{1}, \theta_{2}\right) \Gamma_{0} \psi=-\lim _{z \rightarrow k^{2}+i 0}\left(B_{\theta_{1}, \theta_{2}} q(z, \mathcal{V})-A_{\theta_{1}, \theta_{2}}\right) \Gamma_{0} \psi_{z} \\
& =-\lim _{z \rightarrow k^{2}+i 0}\left(B_{\theta_{1}, \theta_{2}} \Gamma_{1} \gamma(\cdot, z, \mathcal{V})-A_{\theta_{1}, \theta_{2}}\right) \Gamma_{0} \psi_{z} \\
& =-\lim _{z \rightarrow k^{2}+i 0}\left(B_{\theta_{1}, \theta_{2}} \Gamma_{1}-A_{\theta_{1}, \theta_{2}} \Gamma_{0}\right) \psi_{z}=\left(-B_{\theta_{1}, \theta_{2}} \Gamma_{1}+A_{\theta_{1}, \theta_{2}} \Gamma_{0}\right) \psi
\end{aligned}
$$

The $n$-th component of the vector at the 1.h.s. of (2.90) writes as

$$
\begin{aligned}
{\left[\mathcal{M}\left(k, \theta_{1}, \theta_{2}\right) \Gamma_{0}(\phi-\psi)\right]_{n}=\left[-\mathcal{M}\left(k, \theta_{1}, \theta_{2}\right) \Gamma_{0} \psi\right]_{n}=} & \\
& -\sum_{i, j=1}^{4}\left[\left[\mathcal{M}\left(k, \theta_{1}, \theta_{2}\right) \Gamma_{0}(\phi-\psi)\right]_{n} \Gamma_{0} g\left(e_{i}, k, \mathcal{V}\right)\right]_{n}\left[\mathcal{M}^{-1}\left(k, \theta_{1}, \theta_{2}\right) B_{\theta_{1}, \theta_{2}}\right]_{i j}\left[\Gamma_{1} \phi\right]_{j} .
\end{aligned}
$$

Recalling that $\Gamma_{0} g\left(e_{i}, k, \mathcal{V}\right)=e_{i}$, we get

$$
\begin{aligned}
{\left[\mathcal{M}\left(k, \theta_{1}, \theta_{2}\right) \Gamma_{0}(\phi-\psi)\right]_{n} } & = \\
& -\sum_{i, j=1}^{4}\left(\mathcal{M}\left(k, \theta_{1}, \theta_{2}\right)\right)_{n i}\left[\mathcal{M}^{-1}\left(k, \theta_{1}, \theta_{2}\right) B_{\theta_{1}, \theta_{2}}\right]_{i j}\left[\Gamma_{1} \phi\right]_{j}=-\sum_{i, j=1}^{4} B_{n j}\left[\Gamma_{1} \phi\right]_{j}
\end{aligned}
$$

which implies

$$
\mathcal{M}\left(k, \theta_{1}, \theta_{2}\right) \Gamma_{0}(\phi-\psi)=-B \Gamma_{1} \phi .
$$

From (2.90) and (2.91), the interface conditions

$$
A_{\theta_{1}, \theta_{2}} \Gamma_{0} \psi_{-}\left(\cdot, k, \theta_{1}, \theta_{2}\right)=B_{\theta_{1}, \theta_{2}} \Gamma_{1} \psi_{-}\left(\cdot, k, \theta_{1}, \theta_{2}\right),
$$

follow. Since these are equivalent to the ones assigned in the equation (2.73), the function defined in (2.85) is a solution to the problem (2.73), (2.74) -(2.75). The case $k<0$ can be treated by a suitable adaptation of the above arguments.

The regularity of the generalized eigenfunctions w.r.t. to the variables $\left\{x, k, \theta_{1}, \theta_{2}\right\}$ is a consequence of the representation (2.85) and of the properties of the maps $\psi_{-}(\cdot, k), \mathcal{M}^{-1}\left(k, \theta_{1}, \theta_{2}\right), B_{\theta_{1}, \theta_{2}}$, and $g\left(e_{i}, k, \mathcal{V}\right)$ (for this point, we refer to the corresponding definitions and to the results of the Proposition 2.1 and of the Lemmata 2.3 2.4).

As a consequence of the above result, an expansion of $\psi_{-}\left(\cdot, k, \theta_{1}, \theta_{2}\right)$ for small values of $\theta_{i}$ follows.

Corollary 2.7 Let $\psi_{-}\left(\cdot, k, \theta_{1}, \theta_{2}\right)$ denotes a solution to the generalized eigenfunctions problem (2.73), 2.74)-2.75). In the assumptions of the Proposition 2.6, the expansion

$\psi_{-}\left(\cdot, k, \theta_{1}, \theta_{2}\right)-\psi_{-}(\cdot, k)=\mathcal{O}\left(\theta_{2} k\right) G^{\sigma k}(\cdot, b)+\mathcal{O}\left(\frac{\theta_{1} k}{1+|k|}\right) H^{\sigma k}(\cdot, b)+\mathcal{O}\left(\theta_{2} k\right) G^{\sigma k}(\cdot, a)+\mathcal{O}\left(\frac{\theta_{1} k}{1+|k|}\right) H^{\sigma k}(\cdot, a)$.

holds with: $\sigma=\frac{k}{|k|}$. The symbols $\mathcal{O}(\cdot)$ are defined in the sense of the metric space $\mathbb{R} \times \mathcal{B}_{\delta}((0,0))$.

Proof. As already noticed, the assumption of positive potentials (2.50) prevents the Jost's function $w(k)$ to have zeroes on the real axis. In particular, a consequence of the definition (2.76) and of the relations (2.20) is

$$
\psi_{-}(x, k)=\mathcal{O}\left(\frac{k}{1+|k|}\right) ; \quad \partial_{x} \psi_{-}(x, k)=\mathcal{O}(k)
$$

and a direct computation yields

$$
B_{\theta_{1}, \theta_{2}} \Gamma_{1} \psi_{-}(\cdot, k)=\left\{\mathcal{O}\left(\theta_{2} k\right), \mathcal{O}\left(\frac{\theta_{1} k}{1+|k|}\right), \mathcal{O}\left(\theta_{2} k\right), \mathcal{O}\left(\frac{\theta_{1} k}{1+|k|}\right)\right\} .
$$


where the symbols $\mathcal{O}(\cdot)$ are referred to the metric space $\mathbb{R} \times \mathcal{B}_{\delta}((0,0))$. Making use of this expression and of the relations (2.82)-(2.84), we get

$$
\mathcal{M}^{-1}\left(\sigma k, \theta_{1}, \theta_{2}\right) B_{\theta_{1}, \theta_{2}}\left[\Gamma_{1} \psi_{-}(\cdot, k)\right]=\left\{\mathcal{O}\left(\theta_{2} k\right), \mathcal{O}\left(\frac{\theta_{1} k}{1+|k|}\right), \mathcal{O}\left(\theta_{2} k\right), \mathcal{O}\left(\frac{\theta_{1} k}{1+|k|}\right)\right\} .
$$

Then, the expansion (2.93) follows from the formula (2.85) by taking into account (2.96) and the definition (2.77).

\section{Similarity and uniform-in-time estimates for the dynamical system.}

In what follows, $\mathcal{V}$ is a positive short-range potential. With this assumption, $Q_{0,0}(\mathcal{V})$ has a purely absolutely continuous spectrum and the related generalized Fourier transform $\mathcal{F}_{\mathcal{V}}$

$$
\left(\mathcal{F}_{\mathcal{V} \varphi}\right)(k)=\int_{\mathbb{R}} \frac{d x}{(2 \pi)^{1 / 2}} \psi_{-}^{*}(x, k) \varphi(x), \quad \varphi \in L^{2}(\mathbb{R}),
$$

is a unitary map with range $R\left(\mathcal{F}_{\mathcal{V}}\right)=L^{2}(\mathbb{R})$ and an inverse map $\mathcal{F}_{\mathcal{V}}^{-1}$ acting as

$$
\left(\mathcal{F}_{\mathcal{V}}^{-1} f\right)(x)=\int \frac{d k}{(2 \pi)^{1 / 2}} \psi_{-}(x, k) f(k),
$$

for all $f \in L^{2}(\mathbb{R})$. Assume in addition the parameters $\theta_{1}, \theta_{2}$ to be close enough to the origin, so that the expansion (2.93) hold, and consider the operator $\mathcal{W}_{\theta_{1}, \theta_{2}}$ defined by the integral kernel

$$
\mathcal{W}_{\theta_{1}, \theta_{2}}(x, y)=\int_{\mathbb{R}} \frac{d k}{2 \pi} \psi_{-}\left(x, k, \theta_{1}, \theta_{2}\right) \psi_{-}^{*}(y, k) .
$$

The next Proposition shows that $\mathcal{W}_{\theta_{1}, \theta_{2}}$ form an analytic family of bounded operators w.r.t. $\left(\theta_{1}, \theta_{2}\right)$, while, for fixed values of the parameters, $\mathcal{W}_{\theta_{1}, \theta_{2}}$ induces a similarity between $Q_{\theta_{1}, \theta_{2}}(\mathcal{V})$ and $Q_{0,0}(\mathcal{V})$.

Proposition 3.1 Let $\mathcal{V}$ satisfy the conditions (1.9), (2.50) and assume $\left(\theta_{1}, \theta_{2}\right) \in \mathcal{B}_{\delta}((0,0))$ with $\delta>0$ small enough. Then, the set $\left\{\mathcal{W}_{\theta_{1}, \theta_{2}},\left(\theta_{1}, \theta_{2}\right) \in \mathcal{B}_{\delta}((0,0))\right\}$ forms an analytic family of bounded operators in $L^{2}(\mathbb{R})$, w.r.t. $\left(\theta_{1}, \theta_{2}\right)$, and the expansion

$$
\mathcal{W}_{\theta_{1}, \theta_{2}}=1+\mathcal{O}\left(\theta_{1}\right)+\mathcal{O}\left(\theta_{2}\right)
$$

holds in the $\mathcal{L}\left(L^{2}(\mathbb{R}), L^{2}(\mathbb{R})\right)$ operator norm. The couple $Q_{\theta_{1}, \theta_{2}}(\mathcal{V}), Q_{0,0}(\mathcal{V})$ is intertwined through $\mathcal{W}_{\theta_{1}, \theta_{2}}$ by

$$
Q_{\theta_{1}, \theta_{2}}(\mathcal{V}) \mathcal{W}_{\theta_{1}, \theta_{2}}=\mathcal{W}_{\theta_{1}, \theta_{2}} Q_{0,0}(\mathcal{V})
$$

Proof. Let consider the action of $\mathcal{W}_{\theta_{1}, \theta_{2}}$ on $\varphi \in L^{2}(\mathbb{R})$; making use of (3.1) and (3.3), this writes as

$$
\mathcal{W}_{\theta_{1}, \theta_{2}} \varphi=\int_{\mathbb{R}} \frac{d k}{(2 \pi)^{1 / 2}} \psi_{-}\left(\cdot, k, \theta_{1}, \theta_{2}\right)\left(\mathcal{F}_{\mathcal{V}} \varphi\right)(k),
$$

and, expressing $\psi_{-}\left(x, k, \theta_{1}, \theta_{2}\right)$ through the expansion (2.93), we get

$$
\begin{aligned}
\mathcal{W}_{\theta_{1}, \theta_{2}} \varphi & =\int_{\mathbb{R}} \frac{d k}{(2 \pi)^{1 / 2}} \psi_{-}(\cdot, k)\left(\mathcal{F}_{\mathcal{V}} \varphi\right)(k)+\int_{\mathbb{R}} \frac{d k}{(2 \pi)^{1 / 2}}\left[\mathcal{O}\left(\theta_{2} k\right) G^{|k|}(\cdot, b)+\mathcal{O}\left(\theta_{2} k\right) G^{|k|}(\cdot, a)\right]\left(\mathcal{F}_{\mathcal{V}} \varphi\right)(k) \\
& +\int_{\mathbb{R}} \frac{d k}{(2 \pi)^{1 / 2}}\left[\mathcal{O}\left(\frac{\theta_{1} k}{1+|k|}\right) H^{|k|}(\cdot, b)+\mathcal{O}\left(\frac{\theta_{1} k}{1+|k|}\right) H^{|k|}(\cdot, a)\right]\left(\mathcal{F}_{\mathcal{V} \varphi}\right)(k),
\end{aligned}
$$

where, it is important to remark, the symbols $\mathcal{O}(\cdot)$ here denote functions depending only on $k, \theta_{1}$ and $\theta_{2}$, but independent of $x$. Since $\int \frac{d k}{(2 \pi)^{1 / 2}} \psi_{-}(\cdot, k)\left(\mathcal{F}_{\mathcal{V}} \varphi\right)(k)=\mathcal{F}_{\mathcal{V}}^{-1}\left(\mathcal{F}_{\mathcal{V}} \varphi\right)$, this equation yields: $\left(\mathcal{W}_{\theta_{1}, \theta_{2}}-\mathbb{I}\right) \varphi=I+I I$, where

$$
I(\varphi)=\int_{\mathbb{R}} \frac{d k}{(2 \pi)^{1 / 2}}\left[\mathcal{O}\left(\theta_{2} k\right) G^{|k|}(\cdot, b)+\mathcal{O}\left(\theta_{2} k\right) G^{|k|}(\cdot, a)\right]\left(\mathcal{F}_{\mathcal{V}} \varphi\right)(k),
$$


and

$$
I I(\varphi)=\int_{\mathbb{R}} \frac{d k}{(2 \pi)^{1 / 2}}\left[\mathcal{O}\left(\frac{\theta_{1} k}{1+|k|}\right) H^{|k|}(\cdot, b)+\mathcal{O}\left(\frac{\theta_{1} k}{1+|k|}\right) H^{|k|}(\cdot, a)\right]\left(\mathcal{F}_{\mathcal{V} \varphi}\right)(k) .
$$

In order to obtain the expansion (3.4), $L^{2}$-norm estimates of the maps defined in (3.8) and (3.9) are needed. We consider at first the case of $I(\varphi)$; let define $\phi_{\alpha}$ as

$$
\phi_{\alpha}(x)=\int_{\mathbb{R}} \frac{d k}{(2 \pi)^{1 / 2}} \mathcal{O}(k) G^{|k|}(x, \alpha)\left(\mathcal{F}_{\mathcal{V}} \varphi\right)(k), \quad \alpha \in\{a, b\},
$$

being $\mathcal{O}(\cdot)$ depending only on $k$. The $L^{2}$-norm of $\phi_{\alpha}$ is bounded by

$$
\left\|\phi_{\alpha}\right\|_{L^{2}(\mathbb{R})} \leq\left\|1_{\{x \leq a\}} \phi_{\alpha}\right\|_{L^{2}(\mathbb{R})}+\left\|1_{(a, b)} \phi_{\alpha}\right\|_{L^{2}(\mathbb{R})}+\left\|1_{\{x \geq b\}} \phi_{\alpha}\right\|_{L^{2}(\mathbb{R})} .
$$

For $\alpha=b$, making use of the explicit form of $G^{k}(x, b)$, given by (2.45) for $\zeta=k$, and exploiting the relations (2.20) and

$$
1_{\{x \leq a\}} \chi_{-}(x, k)=e^{-i k x}, \quad 1_{\{x \geq b\}} \chi_{+}(x, k)=e^{-i k x},
$$

we have

$$
\begin{aligned}
& 1_{\{x \leq a\}}(x) \mathcal{O}(k) G^{|k|}(x, b)=1_{\{x \leq a\}}(x) \tau_{1}(k) e^{-i|k| x} \\
& 1_{\{x \geq b\}}(x) \mathcal{O}(k) G^{|k|}(x, b)=1_{\{x \geq b\}}(x) \tau_{2}(k) e^{i|k| x}
\end{aligned}
$$

with $\tau_{1}, \tau_{2} \in L_{k}^{\infty}(\mathbb{R})$. In the following, $\mathcal{P}$ denotes be the parity operator: $\mathcal{P} u(t)=u(-t)$; from (3.13), we get

$$
\begin{aligned}
1_{\{x \leq a\}}(x) \phi_{b}(x) & =1_{\{x \leq a\}}(x) \int_{\mathbb{R}} \frac{d k}{(2 \pi)^{1 / 2}} \tau_{1}(k) e^{-i|k| x}\left(\mathcal{F}_{\mathcal{V}} \varphi\right)(k) \\
& =1_{\{x \leq a\}}(x)\left(\mathcal{F}_{0}^{-1}\left(1_{k<0} \tau_{1} \mathcal{F}_{\mathcal{V}} \varphi+\mathcal{P}\left(1_{k>0} \tau_{1} \mathcal{F}_{\mathcal{V}} \varphi\right)\right)\right)(x),
\end{aligned}
$$

where, according to the notation introduced in (3.1), $\mathcal{F}_{0}$ is the standard Fourier transform. Thus, $1_{\{x \leq a\}} \phi_{b}$ is estimated by

$$
\left\|1_{\{x \leq a\}} \phi_{b}\right\|_{L^{2}(\mathbb{R})}=\| \mathcal{F}_{0}^{-1}\left(1_{k<0} \tau_{1} \mathcal{F}_{\mathcal{V} \varphi)}\left\|_{L^{2}(\mathbb{R})}+\right\| \mathcal{F}_{0}^{-1} \mathcal{P}\left(1_{k>0} \tau_{1} \mathcal{F}_{\mathcal{V}} \varphi\right)\left\|_{L^{2}(\mathbb{R})} \lesssim\right\| \varphi \|_{L^{2}(\mathbb{R})},\right.
$$

while, for $1_{\{x \geq b\}} \phi_{b}$, a similar inequality follows by using (3.14)

$$
\left\|1_{\{x \geq b\}} \phi_{b}\right\|_{L^{2}(\mathbb{R})}=\left\|\mathcal{F}_{0}^{-1} \mathcal{P}\left(1_{k<0} \tau_{2} \mathcal{F}_{\mathcal{V}} \varphi\right)\right\|_{L^{2}(\mathbb{R})}+\left\|\mathcal{F}_{0}^{-1}\left(1_{k>0} \tau_{2} \mathcal{F}_{\mathcal{V}} \varphi\right)\right\|_{L^{2}(\mathbb{R})} \lesssim\|\varphi\|_{L^{2}(\mathbb{R})} .
$$

According to definition of $G^{k}(x, b)$ for $x<b$, the term $1_{(a, b)} \phi_{b}$ writes as

$1_{(a, b)}(x) \phi_{b}(x)=1_{(a, b)}(x) \int_{\mathbb{R}} d k \frac{\mathcal{O}(k) \chi_{+}(b,|k|)}{w(|k|)} \chi_{-}(x,|k|)\left(\mathcal{F}_{\mathcal{V}} \varphi\right)(k)=1_{(a, b)}(x) \int_{\mathbb{R}} d k \chi_{-}(x,|k|) \tau_{3}(k)\left(\mathcal{F}_{\mathcal{V}} \varphi\right)(k)$,

where $\tau_{3} \in L_{k}^{\infty}(\mathbb{R})$ is: $\tau_{3}(k)=\frac{\mathcal{O}(k) \chi_{+}(b,|k|)}{w(|k|)}$. Using the definition (2.76) and the identities

$$
\chi_{ \pm}(\cdot,-k)=\chi_{ \pm}^{*}(\cdot, k), \quad w(-k)=w^{*}(k),
$$

it follows

$$
\begin{gathered}
1_{k<0}(k) \chi_{-}(x,-k)=1_{k<0}(k) \frac{w(-k)}{2 i k} \psi_{-}(x, k), \\
1_{k \geq 0}(k) \chi_{-}(x, k)=-1_{k \geq 0}(k) \frac{w(k)}{2 i k} \psi_{-}(x,-k) .
\end{gathered}
$$

Take $\tilde{\tau}_{3}(k)=\tau_{3}(k) \frac{w(-k)}{2 i k}$ and $\hat{\tau}_{3}(k)=\tau_{3}(k) \frac{w(k)}{-2 i k} ;$ it results: $\tilde{\tau}_{3}, \hat{\tau}_{3} \in L_{k}^{\infty}(\mathbb{R})$ and the r.h.s. of (3.18) rephrases as

$$
1_{(a, b)}(x) \phi_{b}(x)=1_{(a, b)}(x)\left[\int_{k<0} d k \psi_{-}(x, k) \tilde{\tau}_{3}(k)\left(\mathcal{F}_{\mathcal{V}} \varphi\right)(k)+\int_{k>0} d k \psi_{-}(x,-k)\left(\hat{\tau}_{3}(k)\left(\mathcal{F}_{\mathcal{V}} \varphi\right)(k)\right)\right] .
$$


The first term identifies with the inverse Fourier transform of $1_{k<0} \tilde{\tau}_{3} \mathcal{F}_{\mathcal{V}} \varphi$,

$$
\int_{k<0} d k \psi_{-}(\cdot, k) \tilde{\tau}_{3}(k)\left(\mathcal{F}_{\mathcal{V}} \varphi\right)(k)=\mathcal{F}_{\mathcal{V}}^{-1}\left(1_{k<0} \tilde{\tau}_{3} \mathcal{F}_{\mathcal{V}} \varphi\right)
$$

while, for the second term, we have

$$
\int_{k>0} d k \psi_{-}(\cdot,-k)\left(\hat{\tau}_{3}(k)\left(\mathcal{F}_{\mathcal{V}} \varphi\right)(k)\right)=-\int_{k<0} d k \psi_{-}(\cdot, k)\left(\mathcal{P}\left(\hat{\tau}_{3} \mathcal{F}_{\mathcal{V}} \varphi\right)\right)(k)=-\mathcal{F}_{\mathcal{V}}^{-1} \mathcal{P}\left(1_{k>0} \hat{\tau}_{3} \mathcal{F}_{\mathcal{V}} \varphi\right) .
$$

The above relations yield the estimate

$$
\left\|1_{(a, b)} \phi_{b}\right\|_{L^{2}(\mathbb{R})}=\left\|1_{(a, b)} \mathcal{F}_{\mathcal{V}}^{-1}\left(1_{k<0} \tilde{\tau}_{3} \mathcal{F}_{\mathcal{V}} \varphi\right)^{*}\right\|_{L^{2}(\mathbb{R})}+\left\|1_{(a, b)} \mathcal{F}_{\mathcal{V}}^{-1} \mathcal{P}\left(1_{k>0} \tilde{\tau}_{3} \mathcal{F}_{\mathcal{V}} \varphi\right)\right\|_{L^{2}(\mathbb{R})} \lesssim\|\varphi\|_{L^{2}(\mathbb{R})}
$$

As a consequence of (3.16), (3.17) and (3.25) we get

$$
\left\|\phi_{b}\right\|_{L^{2}(\mathbb{R})} \lesssim\|\varphi\|_{L^{2}(\mathbb{R})},
$$

and a similar computation in the case of $\phi_{a}$ leads to: $\left\|\phi_{a}\right\|_{L^{2}(\mathbb{R})} \lesssim\|\varphi\|_{L^{2}(\mathbb{R})}$. From the definitions (3.8) and (3.10), it follows

$$
\|I(\varphi)\|_{L^{2}(\mathbb{R})} \lesssim\left|\theta_{2}\right|\left(\left\|\phi_{a}\right\|_{L^{2}(\mathbb{R})}+\left\|\phi_{b}\right\|_{L^{2}(\mathbb{R})}\right) \lesssim\left|\theta_{2}\right|\|\varphi\|_{L^{2}(\mathbb{R})} .
$$

For the map $I I(\varphi)$, we introduce $\psi_{\alpha}$ defined as

$$
\psi_{\alpha}(x)=\int_{\mathbb{R}} d k \mathcal{O}\left(\frac{k}{1+|k|}\right) H^{|k|}(x, \alpha)\left(\mathcal{F}_{\mathcal{V}} \varphi\right)(k), \quad \alpha \in\{a, b\},
$$

where $\mathcal{O}(\cdot)$ depends only on $k$. For $\alpha=b$, the explicit form of $H^{k}(x, b)$, given by (2.46) for $\zeta=k$, and the relations (2.20), (3.12), yield

$$
\begin{aligned}
& 1_{\{x \leq a\}}(x) \mathcal{O}\left(\frac{k}{1+|k|}\right) H^{|k|}(x, b)=1_{\{x \leq a\}}(x) \eta_{1}(k) e^{-i|k| x} \\
& 1_{(a, b)}(x) \mathcal{O}\left(\frac{k}{1+|k|}\right) H^{|k|}(x, b)=1_{(a, b)}(x) \eta_{3}(k) \chi_{-}(x,|k|) \\
& 1_{\{x \geq b\}}(x) \mathcal{O}\left(\frac{k}{1+|k|}\right) H^{|k|}(x, b)=1_{\{x \geq b\}}(x) \eta_{2}(k) e^{i|k| x}
\end{aligned}
$$

where $\eta_{i=1,2,3} \in L_{k}^{\infty}(\mathbb{R})$ are described by $\mathcal{O}\left(\frac{k}{1+|k|}\right)$. Setting: $\tilde{\eta}_{3}(k)=\eta_{3}(k) \frac{w(-k)}{2 i k}$ and $\hat{\eta}_{3}(k)=\eta_{3}(k) \frac{w(k)}{-2 i k}$ (which, according to the characterization of $\eta_{3}$, still implies: $\tilde{\eta}_{3}, \hat{\eta}_{3} \in L_{k}^{\infty}(\mathbb{R})$ ), and proceeding as before, we obtain the decomposition

$$
\begin{aligned}
\psi_{b} & =1_{\{x \leq a\}}\left[\mathcal{F}_{0}^{-1}\left(1_{k<0} \eta_{1} \mathcal{F}_{\mathcal{V} \varphi}+\mathcal{P}\left(1_{k>0} \eta_{1} \mathcal{F}_{\mathcal{V} \varphi}\right)\right)\right] \\
& +1_{\{x \geq b\}}\left[\mathcal{F}_{0}^{-1} \mathcal{P}\left(1_{k<0} \eta_{2} \mathcal{F}_{\mathcal{V} \varphi}+\mathcal{F}_{0}^{-1}\left(1_{k>0} \eta_{2} \mathcal{F}_{\mathcal{V} \varphi}\right]\right]\right. \\
& +1_{(a, b)}\left[\mathcal{F}_{\mathcal{V}}^{-1}\left(1_{k<0} \tilde{n}_{3} \mathcal{F}_{\mathcal{V} \varphi}-\mathcal{F}_{\mathcal{V}}^{-1} \mathcal{P}\left(1_{k>0} \hat{\eta}_{3} \mathcal{F}_{\mathcal{V}} \varphi\right)\right]\right.
\end{aligned}
$$

This entails: $\left\|\psi_{b}\right\|_{L^{2}(\mathbb{R})} \lesssim\|\varphi\|_{L^{2}(\mathbb{R})}$, while, with similar computations, the corresponding estimate in the case of $\psi_{a}$ is obtained. From the definitions (3.9) and (3.28), follows

$$
\|I I\|_{L^{2}(\mathbb{R})} \lesssim\left|\theta_{1}\right|\left(\left\|\psi_{a}\right\|_{L^{2}(\mathbb{R})}+\left\|\psi_{b}\right\|_{L^{2}(\mathbb{R})}\right)
$$

Then, the above estimates imply

$$
\|I I\|_{L^{2}(\mathbb{R})} \lesssim\left|\theta_{1}\right|\|\varphi\|_{L^{2}(\mathbb{R})} .
$$

The expansion (3.4) is a consequence of (3.27) and (3.34). Since the symbols in (3.8)-(3.9) are holomorphic in $\left(\theta_{1}, \theta_{2}\right)$, the operators $\mathcal{W}_{\theta_{1}, \theta_{2}}$ form an analytic family w.r.t. the parameters. 
Next, we consider the relation (3.5)). Let $\varphi \in D\left(Q_{0,0}(\mathcal{V})\right)$, using the functional calculus of $Q_{0,0}(\mathcal{V})$, we have: $\left(\mathcal{F}_{\mathcal{V}}\left(Q_{0,0}(\mathcal{V}) \varphi\right)\right)(k)=k^{2}\left(\mathcal{F}_{\mathcal{V}} \varphi\right)(k)$ and, according to (3.6), the r.h.s. of (3.5) writes as

$$
\mathcal{W}_{\theta_{1}, \theta_{2}} Q_{0,0}(\mathcal{V}) \varphi=\int_{\mathbb{R}} d k \psi_{-}\left(\cdot, k, \theta_{1}, \theta_{2}\right) k^{2}\left(\mathcal{F}_{\mathcal{V}} \varphi\right)(k) .
$$

To discuss the action of $Q_{\theta_{1}, \theta_{2}}(\mathcal{V}) \mathcal{W}_{\theta_{1}, \theta_{2}}$ over $D\left(Q_{0,0}(\mathcal{V})\right)$, we use the expansion

$$
\mathcal{W}_{\theta_{1}, \theta_{2}} \varphi=\varphi+I(\varphi)+I I(\varphi) .
$$

From the above results, the map $I(\varphi)+I I(\varphi)$ can be represented as

$$
\begin{aligned}
I(\varphi)+I I(\varphi) & =1_{\{x \leq a\}}\left[\mathcal{F}_{0}^{-1}\left(1_{k<0} \mu_{1} \mathcal{F}_{\mathcal{V} \varphi}+\mathcal{P}\left(1_{k>0} \mu_{1} \mathcal{F}_{\mathcal{V} \varphi}\right)\right)\right] \\
& +1_{\{x \geq b\}}\left[\mathcal{F}_{0}^{-1} \mathcal{P}\left(1_{k<0} \mu_{2} \mathcal{F}_{\mathcal{V} \varphi}\right)+\mathcal{F}_{0}^{-1}\left(1_{k>0} \mu_{2} \mathcal{F}_{\mathcal{V} \varphi)}\right]\right. \\
& +1_{(a, b)}\left[\mathcal{F}_{\mathcal{V}}^{-1}\left(1_{k<0} \mu_{3} \mathcal{F}_{\mathcal{V} \varphi} \varphi\right) \mathcal{F}_{\mathcal{V}}^{-1}\left(1_{k<0}\left(\mathcal{P}\left(\mu_{4} \mathcal{F}_{\mathcal{V} \varphi)}\right)\right)\right]\right.
\end{aligned}
$$

where $\mu_{i} \in L_{k}^{\infty}(\mathbb{R}), i=1, . .4$, are suitable bounded functions of $k$. Let $u \in L_{k}^{\infty}(\mathbb{R})$ and $\mathcal{V}, \mathcal{V}^{\prime}$ any couple of potentials fulfilling the assumptions; the operators $\mathcal{F}_{\mathcal{V}^{\prime}}^{-1} u \mathcal{F}_{\mathcal{V}}$ and $\mathcal{F}_{\mathcal{V}^{\prime}}^{-1} \mathcal{P} u \mathcal{F}_{\mathcal{V}}$ map $D\left(Q_{0,0}(\mathcal{V})\right)$ into itself. Then, as a consequence of (3.36), (3.37), the operator $\mathcal{W}_{\theta_{1}, \theta_{2}}$ maps $D\left(Q_{0,0}(\mathcal{V})\right)$ into $D(Q(\mathcal{V}))$, while, according to (2.92) and (3.35), $\mathcal{W}_{\theta_{1}, \theta_{2}} \varphi$ fulfills the interface conditions (1.10) for all $\varphi \in D\left(Q_{0,0}(\mathcal{V})\right)$; we obtain: $\mathcal{W}_{\theta_{1}, \theta_{2}} \in \mathcal{L}\left(D\left(Q_{0,0}(\mathcal{V})\right), D\left(Q_{\theta_{1}, \theta_{2}}(\mathcal{V})\right)\right)$. Moreover, from the relation: $\left(Q_{\theta_{1}, \theta_{2}}(\mathcal{V})-k^{2}\right) \psi_{-}\left(\cdot, k, \theta_{1}, \theta_{2}\right)=0$, it follows

$$
Q_{\theta_{1}, \theta_{2}}(\mathcal{V}) \mathcal{W}_{\theta_{1}, \theta_{2}} \varphi=\int_{\mathbb{R}} d k \psi_{-}\left(\cdot, k, \theta_{1}, \theta_{2}\right) k^{2}\left(\mathcal{F}_{\mathcal{V}} \varphi\right)(k),
$$

which leads to (3.5).

\subsection{Proof of the Theorem 1.1.}

When the parameters $\theta_{1}, \theta_{2}$ are chosen in a suitably small neighbourhood of the origin, the expansion (3.4) yields

$$
\mathcal{W}_{\theta_{1}, \theta_{2}}^{-1}=1+\mathcal{O}\left(\theta_{1}\right)+\mathcal{O}\left(\theta_{2}\right)
$$

and $Q_{\theta_{1}, \theta_{2}}(\mathcal{V})$ expresses as the conjugated operator

$$
Q_{\theta_{1}, \theta_{2}}(\mathcal{V})=\mathcal{W}_{\theta_{1}, \theta_{2}} Q_{0,0}(\mathcal{V}) \mathcal{W}_{\theta_{1}, \theta_{2}}^{-1}
$$

Let us introduce

$$
U_{\theta_{1}, \theta_{2}}(t)=\mathcal{W}_{\theta_{1}, \theta_{2}} U_{0,0}(t) \mathcal{W}_{\theta_{1}, \theta_{2}}^{-1}
$$

being $U_{0,0}(t)=e^{-i t Q_{0,0}(\mathcal{V})}$ the unitary propagator associated with $-i Q_{0,0}(\mathcal{V})$. Due to the properties of $\mathcal{W}_{\theta_{1}, \theta_{2}}, U_{\theta_{1}, \theta_{2}}(t)$ is holomorphic w.r.t. $\left(\theta_{1}, \theta_{2}\right)$, while, for fixed values of the parameters, the family $\left\{U_{\theta_{1}, \theta_{2}}(t)\right\}_{t \in \mathbb{R}}$ forms a strongly continuous group on $L^{2}(\mathbb{R})$ and, according to (3.40), (3.41), we have

$$
i \partial_{t}\left(U_{\theta_{1}, \theta_{2}}(t) u\right)=Q_{\theta_{1}, \theta_{2}}(\mathcal{V}) U_{\theta_{1}, \theta_{2}}(t) u
$$

for all $u \in L^{2}(\mathbb{R})$. This allows to identify $U_{\theta_{1}, \theta_{2}}(t)$ with the quantum dynamical system generated by $-i Q_{\theta_{1}, \theta_{2}}(\mathcal{V})$. Making use of (3.4) and (3.39), we get

$$
U_{\theta_{1}, \theta_{2}}(t)=U_{0,0}(t)+\mathcal{R}\left(t, \theta_{1}, \theta_{2}\right),
$$

where the remainder term is strongly continuous and uniformly bounded w.r.t. $t$ in the $L^{2}$-operator norm, allowing the representation: $\mathcal{R}\left(t, \theta_{1}, \theta_{2}\right)=\mathcal{O}\left(\theta_{1}\right)+\mathcal{O}\left(\theta_{2}\right)$.

\subsection{Time dependent wave operators and scattering systems.}

So far, we have investigated the continuity of the dynamical system generated by $-i Q_{\theta_{1}, \theta_{2}}(\mathcal{V})$ w.r.t. the parameters $\theta_{i=1,2}$. This has been analyzed by using small $\theta_{i}$ expansions of the 'stationary wave operators' $\mathcal{W}_{\theta_{1}, \theta_{2}}$ defined in (3.3). In what follows we consider the scattering problem for the pair $\left\{Q_{\theta_{1}, \theta_{2}}(\mathcal{V}), Q_{0,0}(\mathcal{V})\right\}$ and show that $\mathcal{W}_{\theta_{1}, \theta_{2}}$ coincides with a wave operator of this couple. The next Lemma discusses this point under the assumptions of the Proposition 3.1 . 
Lemma 3.2 Let $\mathcal{V}$ fulfills the conditions (1.9), (2.50) and assume $\left(\theta_{1}, \theta_{2}\right) \in \mathcal{B}_{\delta}((0,0))$ with $\delta>0$ small enough. Then

$$
\mathrm{S}-\lim _{t \rightarrow-\infty} e^{i t Q_{\theta_{1}, \theta_{2}}(\mathcal{V})} e^{-i t Q_{0,0}(\mathcal{V})}=\mathcal{W}_{\theta_{1}, \theta_{2}}
$$

Proof. Let introduce the modified transform $\mathcal{F}_{\mathcal{V}, \theta_{1}, \theta_{2}}$ defined by

$$
\mathcal{F}_{\mathcal{V}, \theta_{1}, \theta_{2}}^{-1} f=\int_{\mathbb{R}} d k \psi_{-}\left(x, k, \theta_{1}, \theta_{2}\right) f(k), \quad f \in L^{2}(\mathbb{R}) .
$$

The action of $\mathcal{W}_{\theta_{1}, \theta_{2}}$ can be expressed in terms of $\mathcal{F}_{\mathcal{V}, \theta_{1}, \theta_{2}}$ and $\mathcal{F}_{\mathcal{V}}$ as $\mathcal{W}_{\theta_{1}, \theta_{2}}=\mathcal{F}_{\mathcal{V}, \theta_{1}, \theta_{2}}^{-1} \mathcal{F}_{\mathcal{V}}$, from which we get: $\mathcal{F}_{\mathcal{V}, \theta_{1}, \theta_{2}}=\mathcal{F}_{\mathcal{V}} \mathcal{W}_{\theta_{1}, \theta_{2}}^{-1}$. Due to the expansion (3.4), it results

$$
\mathcal{F}_{\mathcal{V}, \theta_{1}, \theta_{2}}=\mathcal{F}_{\mathcal{V}}\left(1+\mathcal{O}\left(\theta_{1}\right)+\mathcal{O}\left(\theta_{2}\right)\right)
$$

in the $L^{2}$-operator norm sense. Making use of the intertwining property, we have

$$
\mathcal{W}_{\theta_{1}, \theta_{2}}^{*}\left(Q_{\theta_{1}, \theta_{2}}(\mathcal{V})\right)^{*}=Q_{0,0}(\mathcal{V}) \mathcal{W}_{\theta_{1}, \theta_{2}}^{*} .
$$

Since $\mathcal{W}_{\theta_{1}, \theta_{2}}^{*}=\mathcal{F}_{\mathcal{V}}^{-1}\left(\mathcal{F}_{\mathcal{V}, \theta_{1}, \theta_{2}}^{-1}\right)^{*}$ and $\left(Q_{\theta_{1}, \theta_{2}}(\mathcal{V})\right)^{*}=Q_{-\theta_{2}^{*},-\theta_{1}^{*}}(\mathcal{V})$ (see eq. (1.12) $)$, it follows

$$
\mathcal{F}_{\mathcal{V}}^{-1}\left(\mathcal{F}_{\mathcal{V}, \theta_{1}, \theta_{2}}^{-1}\right)^{*} Q_{-\theta_{2}^{*},-\theta_{1}^{*}}(\mathcal{V})=Q_{0,0}(\mathcal{V}) \mathcal{F}_{\mathcal{V}}^{-1}\left(\mathcal{F}_{\mathcal{V}, \theta_{1}, \theta_{2}}^{-1}\right)^{*} .
$$

Let us denote with $A$ the operator of multiplication by $k^{2}$. Using the functional calculus for $Q_{0,0}(\mathcal{V})$, this operator is represented by: $A=\mathcal{F}_{\mathcal{V}} Q_{0,0}(\mathcal{V}) \mathcal{F}_{\mathcal{V}}^{-1}$, and the previous relation rephrases as

$$
\left(\mathcal{F}_{\mathcal{V}, \theta_{1}, \theta_{2}}^{-1}\right)^{*} Q_{-\theta_{2}^{*},-\theta_{1}^{*}}(\mathcal{V})=A\left(\mathcal{F}_{\mathcal{V}, \theta_{1}, \theta_{2}}^{-1}\right)^{*} .
$$

Then, taking the adjoint, yields

$$
Q_{\theta_{1}, \theta_{2}}(\mathcal{V}) \mathcal{F}_{\mathcal{V}, \theta_{1}, \theta_{2}}^{-1}=\mathcal{F}_{\mathcal{V}, \theta_{1}, \theta_{2}}^{-1} A .
$$

To identify $\mathcal{W}_{\theta_{1}, \theta_{2}}$ with the wave operator, according to the time dependent definition

$$
W_{-}\left(Q_{\theta_{1}, \theta_{2}}(\mathcal{V}), Q_{0,0}(\mathcal{V})\right)=\mathrm{s}^{-} \lim _{t \rightarrow-\infty} e^{i t Q_{\theta_{1}, \theta_{2}}(\mathcal{V})} e^{-i t Q_{0,0}(\mathcal{V})},
$$

it is enough to prove that

$$
\lim _{t \rightarrow-\infty}\left\|\left(e^{i t Q_{\theta_{1}, \theta_{2}}(\mathcal{V})} e^{-i t Q_{0,0}(\mathcal{V})}-\mathcal{W}_{\theta_{1}, \theta_{2}}\right) u\right\|_{L^{2}(\mathbb{R})}=0 .
$$

Explicitly, the function in (3.52) reads as

$$
\left(e^{i t Q_{\theta_{1}, \theta_{2}}(\mathcal{V})} e^{-i t Q_{0,0}(\mathcal{V})}-\mathcal{F}_{\mathcal{V}, \theta_{1}, \theta_{2}}^{-1} \mathcal{F}_{\mathcal{V}}\right) u .
$$

Setting $g=\mathcal{F}_{\mathcal{V}} u$, we have: $e^{-i t Q_{0,0}(\mathcal{V})} u=\mathcal{F}_{\mathcal{V}}^{-1} e^{-i t A} g$, and (3.53) rephrases as

$$
e^{i t Q_{\theta_{1}, \theta_{2}}(\mathcal{V})}\left(\mathcal{F}_{\mathcal{V}}^{-1} e^{-i t A}-e^{-i t Q_{\theta_{1}, \theta_{2}}(\mathcal{V})} \mathcal{F}_{\mathcal{V}, \theta_{1}, \theta_{2}}^{-1}\right) g .
$$

Then, using (3.50) and the definitions (3.1), (3.45), we get

$$
\begin{aligned}
& \left(e^{i t Q_{\theta_{1}, \theta_{2}}(\mathcal{V})} e^{-i t Q_{0,0}(\mathcal{V})}-\mathcal{W}_{\theta_{1}, \theta_{2}}\right) u=e^{i t Q_{\theta_{1}, \theta_{2}}(\mathcal{V})}\left(\mathcal{F}_{\mathcal{V}}^{-1}-\mathcal{F}_{\mathcal{V}, \theta_{1}, \theta_{2}}^{-1}\right) e^{-i t A} g \\
& =e^{i t Q_{\theta_{1}, \theta_{2}}(\mathcal{V})} \int_{\mathbb{R}} d k\left(\psi_{-}(\cdot, k)-\psi_{-}\left(\cdot, k, \theta_{1}, \theta_{2}\right)\right) e^{-i t k^{2}} g(k), \quad g \in \mathcal{F}_{\mathcal{V}} u .
\end{aligned}
$$

Under our assumptions, the result of the Corollary 2.7 applies and the r.h.s. of (3.55) can be further developed through the expansion (2.93). This yields

$$
\begin{aligned}
& \left(e^{i t Q_{\theta_{1}, \theta_{2}}(\mathcal{V})} e^{-i t Q_{0,0}(\mathcal{V})}-\mathcal{W}_{\theta_{1}, \theta_{2}}\right) u= \\
& \quad \int_{\mathbb{R}} d k \mathcal{O}\left(\theta_{2} k\right) G^{\sigma k}(\cdot, b) e^{-i t k^{2}} g(k)+\int_{\mathbb{R}} d k \mathcal{O}\left(\frac{\theta_{1} k}{1+|k|}\right) H^{\sigma k}(\cdot, b) e^{-i t k^{2}} g(k) \\
& +\int_{\mathbb{R}} d k \mathcal{O}\left(\theta_{2} k\right) G^{\sigma k}(\cdot, a) e^{-i t k^{2}} g(k)+\int_{\mathbb{R}} d k \mathcal{O}\left(\frac{\theta_{1} k}{1+|k|}\right) H^{\sigma k}(\cdot, a) e^{-i t k^{2}} g(k) .
\end{aligned}
$$


where $\sigma=\frac{k}{|k|}$, while the functions $\mathcal{O}\left(\theta_{2} k\right)$ and $\mathcal{O}\left(\frac{\theta_{1} k}{1+|k|}\right)$ are independent of $x$. To obtain (3.44), it is enough to show that, for all $g \in \mathcal{C}_{0}^{\infty}(\mathbb{R})$, limits of the type (3.52) are available for each term at the r.h.s. of (3.56). In what follows, we consider the first contribution of (3.56), while the other terms can be treated within the same approach. Since we work with fixed $\left(\theta_{1}, \theta_{2}\right)$, the dependence from these parameters is next omitted. Our aim is to prove

$$
\lim _{|t| \rightarrow \infty}\left\|\int_{\mathbb{R}} d k \mathcal{O}(k) G^{\sigma k}(\cdot, b) e^{-i t k^{2}} g(k)\right\|_{L^{2}(\mathbb{R})}=0,
$$

when $g \in \mathcal{C}_{0}^{\infty}(\mathbb{R})$. We use the definitions (2.45), (2.51), with $\zeta=k$, to write

$$
\left\|\int_{\mathbb{R}} d k \mathcal{O}(k) G^{\sigma k}(\cdot, b) e^{-i t k^{2}} g(k)\right\|_{L^{2}(\mathbb{R})}=I+I I
$$

where

$$
\begin{gathered}
I=\int_{-\infty}^{b} d x\left|\int_{\mathbb{R}} d k \frac{\mathcal{O}(k)}{w(|k|)} \chi_{-}(x,|k|) \chi_{+}(b,|k|) e^{-i t k^{2}} g(k)\right|^{2}, \\
I I=\int_{b}^{+\infty} d x\left|\int_{\mathbb{R}} d k \frac{\mathcal{O}(k)}{w(|k|)} \chi_{+}(x,|k|) \chi_{-}(b,|k|) e^{-i t k^{2}} g(k)\right|^{2} .
\end{gathered}
$$

Recall that $\chi_{ \pm}$are defined through the equations

$$
\begin{aligned}
& 1_{x<b}(x) \chi_{+}(x, k)=e^{i k x}+\int_{x}^{b} \frac{\sin k(t-x)}{k} \mathcal{V}(t) \chi_{+}(t, k) d t, \quad 1_{x \geq b}(x) \chi_{+}(x, k)=e^{i k x}, \\
& 1_{x>a}(x) \chi_{-}(x, k)=e^{-i k x}-\int_{a}^{x} \frac{\sin k(t-x)}{k} \mathcal{V}(t) \chi_{-}(t, k) d t, \quad 1_{x \leq a}(x) \chi_{-}(x, k)=e^{-i k x},
\end{aligned}
$$

and introduce the functions

$$
\gamma_{+}(x)=\int_{x}^{b}|\mathcal{V}(t)| d t, \quad \gamma_{-}(x)=\int_{a}^{x}|\mathcal{V}(t)| d t
$$

For compactly supported short-range potentials (see (11.9) ) , it results: $\gamma_{+} \in L^{2}((a,+\infty))$ and $\gamma_{-} \in$ $L^{2}((-\infty, b))$. As stated in the Proposition 2.1] $\chi_{ \pm}$are uniformly bounded w.r.t. $x, k \in \mathbb{R}$ and the relations (3.60), (3.61) rephrase as

$$
\begin{array}{cc}
1_{x<b}(x) \chi_{+}(x, k)=e^{i k x}+\ell_{+}(x, k), & 1_{x \geq b}(x) \chi_{+}(x, k)=e^{i k x}, \\
1_{x>a}(x) \chi_{-}(x, k)=e^{-i k x}+\ell_{-}(x, k), & 1_{x \leq a}(x) \chi_{-}(x, k)=e^{-i k x},
\end{array}
$$

with $\ell_{ \pm}$s.t.:

$$
\left|\ell_{ \pm}(x, k)\right|<\frac{1}{k}\left\|\chi_{ \pm}\right\|_{L_{x, k}^{\infty}\left(\mathbb{R}^{2}\right)} \gamma_{ \pm}(x) .
$$

Plugging these relations into (3.58)-(3.59) and using $1_{x>b}(x) g_{+}(x, k)=0$, we get

$$
\begin{aligned}
I & \leq \int_{-\infty}^{b} d x\left|\int_{\mathbb{R}} d k e^{-i|k| x} e^{-i t k^{2}} \frac{\mathcal{O}(k) e^{i|k| b}}{w(|k|)} g(k)\right|^{2}+\int_{-\infty}^{b} d x\left|\int_{\mathbb{R}} d k \ell_{-}(x,|k|) e^{-i t k^{2}} \frac{\mathcal{O}(k) e^{i|k| b}}{w(|k|)} g(k)\right|^{2} \\
I I & =\int_{b}^{+\infty} d x\left|\int_{\mathbb{R}} d k e^{i|k| x} e^{-i t k^{2}} \frac{\mathcal{O}(k)}{w(|k|)} \chi_{-}(b,|k|) g(k)\right|^{2}
\end{aligned}
$$


With the change of variable: $s=-x+b$, the first integral at the r.h.s. of (3.66) writes as

$$
\int_{-\infty}^{b} d x\left|\int_{\mathbb{R}} d k e^{-i|k| x} e^{-i t k^{2}} \frac{\mathcal{O}(k) e^{i|k| b}}{w(|k|)} g(k)\right|^{2}=\int_{0}^{+\infty} d s\left|\int_{\mathbb{R}} d k e^{i|k| s-i t k^{2}}\left(\frac{\mathcal{O}(k)}{w(|k|)} g(k)\right)\right|^{2},
$$

while, setting: $s=x-b$, the identity (3.67) rephrases as

$$
I I=\int_{0}^{+\infty} d s\left|\int_{\mathbb{R}} d k e^{i|k| s} e^{-i t k^{2}}\left(\frac{\mathcal{O}(k) e^{i|k| b}}{w(|k|)} \chi_{-}(b,|k|) g(k)\right)\right|^{2} .
$$

Due to the relation $w(k)=\mathcal{O}(1+|k|)$ (see the proof of Lemma 2.3 $)$, the above appearing functions,

$$
\frac{\mathcal{O}(k)}{w(k)} g(k) \quad \text { and } \quad \chi_{-}(b,|k|) g(k)
$$

both belong to $L_{k}^{2}(\mathbb{R})$. Moreover, as a consequence of (3.65), it results

$$
\left|\ell_{-}(x,|k|) \frac{\mathcal{O}(k) e^{i|k| b}}{w(|k|)}\right| \leq\left\|\chi_{-}\right\|_{L_{x, k}^{\infty}\left(\mathbb{R}^{2}\right)}\left|\frac{\mathcal{O}(k)}{k w(|k|)}\right| \gamma_{-}(x) \lesssim \gamma_{-}(x) \in L^{2}((-\infty, b)),
$$

We get

$$
\begin{aligned}
& I \leq \int_{0}^{+\infty} d s\left|\int_{\mathbb{R}} d k e^{i|k| s-i t k^{2}} q_{1}(k)\right|^{2}+\int_{-\infty}^{b} d x\left|\int_{\mathbb{R}} d k e^{-i t k^{2}} q_{2}(k, x) g(k)\right|^{2}, \\
& I I=\int_{0}^{+\infty} d s\left|\int_{\mathbb{R}} d k e^{i|k| s-i t k^{2}} q_{3}(k)\right|^{2} .
\end{aligned}
$$

where, according to the previous remarks, $q_{1}, q_{3} \in L_{k}^{2}(\mathbb{R})$, while $q_{2}$ allows the estimate

$$
\left|q_{2}(k, x)\right| \leq f(x) \in L^{2}((-\infty, b))
$$

Then, it follows from an application of the Lemma 2.6.4 of [32] that

$$
\lim _{t \rightarrow-\infty} \int_{0}^{+\infty} d s\left|\int_{\mathbb{R}} d k e^{i|k| s-i t k^{2}} q_{j}(k)\right|^{2}=0, \quad j=1,3,
$$

Moreover, for $g \in \mathcal{C}_{0}^{\infty}(\mathbb{R})$, the Riemann-Lebesgue Lemma implies

$$
\lim _{|t| \rightarrow \infty} \int_{\mathbb{R}} d k e^{-i t k^{2}} q_{2}(k, x) g(k)=0 ;
$$

thus, using the estimate

$$
\left|\int_{\mathbb{R}} d k e^{-i t k^{2}} q_{2}(k, x) g(k)\right| \lesssim|f(x)|\left(\int_{\mathbb{R}} d k|g(k)|\right) \lesssim|f(x)|,
$$

and the dominated convergence theorem, we get

$$
\lim _{|t| \rightarrow \infty}\left\|\int_{\mathbb{R}} d k e^{-i t k^{2}} q_{2}(k, x) g(k)\right\|_{L^{2}(-\infty, b)}=0 .
$$

The above result exploits the condition: $\left(\theta_{1}, \theta_{2}\right) \in \mathcal{B}_{\delta}((0,0))$ which has been previously introduced to identify the spectra of the operators $Q_{\theta_{1}, \theta_{2}}(\mathcal{V})$ and $Q_{0,0}(\mathcal{V})$, and to compare the corresponding quantum dynamics. Nevertheless a question is left open: is a small parameter condition necessary in order that the pair $\left\{Q_{\theta_{1}, \theta_{2}}(\mathcal{V}), Q_{0,0}(\mathcal{V})\right\}$ forms a complete scattering system ? Actually this restriction does not seems to be necessary. It has been shown, indeed, that a key point in the development of the scattering theory for the possibly non-selfadjoint pair $\left\{H_{0}, H_{1}\right\}$ is the existence of the strong limit on the real axis of the characteristic functions associated with $H_{i=0,1}$ (e.g. in [29] and [30]). In particular, the Theorem 4.1 in 29] makes use of this assumption to study the existence of the related wave operators. 
According to [28, the resolvent formula (2.16) implies that, for any $\left(\theta_{1}, \theta_{2}\right)$, the characteristic function of the operator $Q_{\theta_{1}, \theta_{2}}(\mathcal{V})$ has boundary values a.e. on the real axis (for this point we refer to the last Proposition in [28] and to the references therein). This suggests the possibility of defining the scattering system $\left\{Q_{\theta_{1}, \theta_{2}}(\mathcal{V}), Q_{0,0}(\mathcal{V})\right\}$ without restrictions on $\left(\theta_{1}, \theta_{2}\right)$.

A slightly different approach to the scattering problem consists in characterizing the scattering matrix for $\left\{Q_{\theta_{1}, \theta_{2}}(\mathcal{V}), Q_{0,0}(\mathcal{V})\right\}$. In the case of selfadjoint extensions of a symmetric operator, a relation between the scattering matrix and the Weyl function, associated with a boundary triple, have been established in [4, while extensions of results from 4] to certain non-selfadjoint situations (dissipative/accumulative) have been presented in, [3], 5]. A generalization to the case of $Q_{\theta_{1}, \theta_{2}}(\mathcal{V})$ would represents a useful insight in the study of the scattering properties of the system $\left\{Q_{\theta_{1}, \theta_{2}}(\mathcal{V}), Q_{0,0}(\mathcal{V})\right\}$.

\section{Further perspectives: the regime of quantum wells in a semi- classical island.}

Let introduce the modified operators $Q_{\theta_{1}, \theta_{2}}^{h}(\mathcal{V})$, depending on the small parameter $h \in\left(0, h_{0}\right), h_{0}>0$, and defined according to

$$
Q_{\theta_{1}, \theta_{2}}^{h}(\mathcal{V}):\left\{\begin{array}{l}
D\left(Q_{\theta_{1}, \theta_{2}}^{h}(\mathcal{V})\right)=\left\{u \in H^{2}(\mathbb{R} \backslash\{a, b\}) \mid \text { (1.10) holds }\right\}, \\
\left(Q_{\theta_{1}, \theta_{2}}^{h}\left(\mathcal{V}^{h}\right) u\right)(x)=-h^{2} u^{\prime \prime}(x)+\mathcal{V}^{h}(x) u(x), \quad x \in \mathbb{R} \backslash\{a, b\} .
\end{array}\right.
$$

with $\mathcal{V}^{h} h$-dependent and locally supported on $[a, b]$. In the applications perspectives, rather relevant is the case of a positive and bounded $\mathcal{V}^{h}$ formed by the superposition of a potential barrier and a collection of potential wells supported on a region of size $h$ (these are usually referred to as quantum wells). Hamiltonians of this type have been introduced in [12, where the case: $\theta_{2}=3 \theta_{1}$ is considered, with the purpose of realizing models of electronic transverse transport through resonant heterostructures. When the initial state describes incoming charge carriers in the conduction band, the quantum dynamics of such systems is expected be driven by the, possibly non-linear, adiabatic evolution of a finite number of resonant states related to the shape resonances. This picture, arising in the physical literature, is confirmed by the analysis presented in [16], 25] concerning the case of a 1D Schrödinger-Poisson selfadjoint model with a double barrier, and in [13], where an application involving Hamiltonians of the type $Q_{\theta_{1}, \theta_{2}}^{h}(\mathcal{V})$ is considered.

As previously remarked, the artificial interface conditions allow to develop an alternative approach to the adiabatic evolution for shape resonances. In the particular case where $\theta_{2}=3 \theta_{1}$, using suitable exterior dilations, it is possible to write the evolution problem for the resonant states of the modified operators $Q_{\theta_{1}, \theta_{2}}^{h}(\mathcal{V})$ as a dynamical system of contractions. Then the adiabatic approximations can be obtained by using a 'standard' approach and reasonably weak assumptions on the regularity-in-time of the potential, as it has been shown in [12, while a similar strategy would not work in the selfadjoint case, due to the lack of accretivity of the corresponding complex deformed operator. This justifies the interest in the operators $Q_{\theta_{1}, \theta_{2}}^{h}(\mathcal{V})$ as models for the electronic quantum transport in the regime of quantum wells in a semiclassical island, and motivates an extension of the previous analysis taking into account the role of the scaling parameter $h$ in the definition of the modified dynamics. Our aim is to obtain, in the $h$-dependent case, a comparison between the dynamical system modified by non-mixed interface conditions and the unitary dynamics generated by the selfadjoint Hamiltonians $Q_{0,0}^{h}\left(\mathcal{V}^{h}\right)$. Proceeding in this direction, a first step consists in the study of the Jost's solutions, the generalized eigenfunctions and the Green's kernel associated to operators of the type $Q_{0,0}^{h}(\mathcal{V})$, which, according to the definition (4.1), is given by

$$
Q_{0,0}^{h}(\mathcal{V}):\left\{\begin{array}{l}
D\left(Q_{0,0}^{h}(\mathcal{V})\right)=H^{2}(\mathbb{R}), \\
\left(Q_{0,0}^{h}(\mathcal{V}) u\right)(x)=-h^{2} u^{\prime \prime}(x)+\mathcal{V}(x) u(x), \quad x \in \mathbb{R},
\end{array}\right.
$$

with $\mathcal{V}$, possibly depending on $h$, locally supported on $[a, b]$. In what follows, $\chi_{ \pm}^{h}(\cdot, \zeta, \mathcal{V})$ denote the solutions of the equation

$$
\left(-h^{2} \partial_{x}^{2}+\mathcal{V}\right) u=\zeta^{2} u
$$

fulfilling the conditions

$$
\left.\chi_{+}^{h}(\cdot, \zeta, \mathcal{V})\right|_{x>b}=e^{i \frac{\zeta}{h} x},\left.\quad \chi_{-}^{h}(\cdot, \zeta, \mathcal{V})\right|_{x<a}=e^{-i \frac{\zeta}{h} x}
$$


It is worthwhile to notice that, in the attempt of extending our approach to this new setting, all the estimates involved in the proofs depend on $h$ and exhibit exponential bounds w.r.t. to the small parameter. To fix this point, let $h>0$ and consider the functions $\chi_{ \pm}^{h}$. The rescaled functions $b_{ \pm}^{h}(x, \zeta)=e^{\mp i \frac{\zeta}{h} x} \chi_{ \pm}^{h}(x, \zeta)$ are defined through a Picard iteration procedure (see the e.g. in the eq. $(2.25)$ ). Taking into account the small- $h$ behviour of the corresponding rescaled kernels, it results

$$
\sup _{x \in \mathbb{R}, \zeta \in \overline{\mathbb{C}^{+}}}\left|b_{ \pm}(x, \zeta)\right| \leq e^{\frac{C_{0}}{h^{2}}}, \quad \sup _{x \in \mathbb{R}, \zeta \in \overline{\mathbb{C}^{+}}}\left|b_{ \pm}^{\prime}(x, \zeta)\right| \leq e^{\frac{C_{1}}{h^{2}}}
$$

where the coefficients $C_{i}, i=0,1$, possibly depend on the data $a, b$, and $\|\mathcal{V}\|_{L^{1}(a, b)}$. Then, a suitable rewriting of the Krein's formula (2.16) and of the results of the Lemmata 2.3 and 2.4 in the $h$-dependent case would allows to express the $h$-dependent wave operators as: $\mathcal{W}_{\theta_{1}, \theta_{2}}^{h}=1+\mathcal{O}(h)+\mathcal{O}(h)$, provided that

$$
\left(\theta_{1}, \theta_{2}\right) \in \mathcal{B}_{\rho(h)}((0,0)), \quad \text { with } \rho(h)=h e^{-\frac{\tilde{\sigma}}{h^{2}}}
$$

and $\tilde{C}>0$ large enough.

Operators defined with the prescription (4.6) appear to be of small interest in the applications perspective. At this concern we recall that the adiabatic theorem obtained in [12] applies with: $\theta_{i}=c_{i} h^{N_{0}}$, $i=1,2$, for some $N_{0} \in \mathbb{N}$ (see Theorem 7.1 in [12]). In this connection, it is important to relax the constraint expressed by (4.6) in order to obtain small- $h$ expansions of the waves operators, holding at least in a suitable subspace of $L^{2}$, when the parameters are assumed to be only polynomially smalls w.r.t. $h$.

According to formulas of Section 2.3, the key to obtain small- $\theta_{i=1,2}$ expansions of the generalized eigenfunctions, and then of the wave operators, consists in controlling the boundary values of Green's functions as $z$ approaches the continuous spectrum. Introducing quantum wells in the model, produces resonances with exponentially small imaginary parts as $h \rightarrow 0$. This means that, the Green's functions, which are expressed in terms of the Jost solutions and the Jost function, will be exponentially large w.r.t. $h$ somewhere in the potential structure when $z$ is close to the corresponding energies. Nevertheless, their values on the boundary of the potential support are expected to be, at most, of order $\mathcal{O}\left(\frac{1}{h^{N_{0}}}\right)$ for a suitable $N_{0} \in \mathbb{N}$; an explicit example of this mechanism can be found in 13. Studying the Green function around a resonant energy requires the introduction of a Dirichlet problem in order to resolve the spectral singularity and to match the complete problem with some combination of this spectral problem with the filled wells spectral problem. Following [15, 14, the Grushin technique can be used for handling this matching and obtain resolvent approximations. Developing this approach is a further perspective of our work.

Akcnowledgement This work arises from a question addressed by A. Teta and has largely profited from useful discussions with F. Nier and C.A. Pillet. The author is also indebted to S. Naboko and H. Neidhardt for their important remarks.

\section{References}

[1] J. Aguilar, J.M. Combes. A class of analytic perturbations for one-body Schrödinger Hamiltonians. Comm. Math. Phys., 22, 269-279, 1971.

[2] E. Balslev, J.M. Combes. Spectral properties of many-body Schrödinger operators with dilatationanalytic interactions. Comm. Math. Phys., 22, 280-294, 1971.

[3] J. Behrndt, M.M. Malamud, H. Neidhardt. Finite rank perturbations, scattering matrices and inverse problems. In: Recent advances in operator theory in Hilbert and Krein spaces, Oper. Theory Adv. Appl., 198, 61-85, Birkhäuser Verlag, Basel, 2010.

[4] J. Behrndt, M.M. Malamud, H. Neidhardt. Scattering matrices and Weyl functions. Proc. Lond. Math. Soc. 97, no.3, 568-598, 2008.

[5] J. Behrndt, M.M. Malamud, H. Neidhardt. Scattering theory for open quantum systems with finite rank coupling. Math. Phys. Anal. Geom., 10 no.4, 313-358, 2007. 
[6] V. Bonnaillie-Noël, F. Nier, Y. Patel. Far from equlibrium steady states of 1D-Schrödinger-Poisson systems with quantum wells I. Ann. I.H.P. An. Non Linéaire, 25, 937-968, 2008.

[7] V. Bonnaillie-Noël, F. Nier, Y. Patel. Far from equlibrium steady states of 1D-Schrödinger-Poisson systems with quantum wells II. J. Math. Soc. of Japan., 61, 65-106, 2009.

[8] V. Bonnaillie-Noël, F. Nier, Y. Patel. Computing the steady states for an asymptotic model of quantum transport in resonant heterostructures. Journal of Computational Physics, 219(2), 644670, 2006.

[9] M. Brown, M. Marletta, S. Naboko, I. Wood. Boundary triplets and M-functions for non-selfadjoint operators, with applications to elliptic PDEs and block operator matrices. J. Lond. Math. Soc. (2) 77 no. 3, 700-718, 2008.

[10] J. Brüning, V.Geyler, K. Pankrashkin. Spectra of self-adjoint extensions and applications to solvable Schrödinger operators, Rev. Math. Phys., 20 no.1, 1-70, 2008.

[11] V.A. Derkach, M. M. Malamud. Generalized resolvents and the boundary value problems for Hermitian operators with gaps, J. Funct. Anal. 95, 1-95, 1991.

[12] A. Faraj, A. Mantile, F. Nier. Adiabatic evolution of 1D shape resonances: an artificial interface conditions approach. M3AS, 21 no. 3, 541-618, 2011.

[13] A. Faraj, A. Mantile, F. Nier. An explicit model for the adiabatic evolution of quantum observables driven by 1D shape resonances. J. Phys. A: Math. Theor. 43, 2010.

[14] B. Helffer. Semiclassical analysis for the Schrödinger operator and applications, volume 1336 of Lecture Notes in Mathematics. Springer-Verlag, Berlin, 1988.

[15] B. Helffer, J. Sjöstrand. Résonances en limite semi-classique. Mém. Soc. Mat. France (N.S.), number 24-25, 1986.

[16] G. Jona-Lasionio, C. Presilla, J. Sjöstrand. On the Schrödinger equation with concentrated non linearities. Ann. Physics, 240 no.1, 1-21,1995.

[17] T. Kato. Wave Operators and Similarity for Some Non-Selfadjoint Operators. Math. Annalen, 162, 258-279, 1966.

[18] D. Krejčiř́ík, P. Siegl, J. Železný. On the similarity of Sturm-Liouville operators with non-Hermitian boundary conditions to self-adjoint and normal operators. To appear on Complex Anal. Oper. Theory. Preprint: arXiv:1108.4946v1.

[19] V.E. Lyantze, O.G. Storozh. Methods of the theory of unbounded operators. Naukova Dumka, Kiev, 1983.

[20] M.M. Malamud, V.I. Mogilevskii. On extensions of dual pairs of operators. Dopov. Nats. Akad. Nauk Ukr. Mat. Prirodozn. Tekh. Nauki, no. 1, 30-37, 1997.

[21] M.M. Malamud, V.I. Mogilevskii. On Weyl functions and Q-functions of dual pairs of linear relations. Dopov. Nats. Akad. Nauk Ukr. Mat. Prirodozn. Tekh. Nauki, no. 4, 32-37, 1999.

[22] M.M. Malamud, V.I. Mogilevskii. Kreı̆n type formula for canonical resolvents of dual pairs of linear relations. Methods Funct. Anal. Topology, 8, no. 4, 72-100, 2002.

[23] G. Nenciu, Linear adiabatic theory. Exponential estimates. Comm. Math.Phys.,152(3); 479-496, 1993.

[24] K. Pankrashkin, Resolvents of self-adjoint extensions with mixed boundary conditions. Rep. Math. Phys., 58(2), 207-221, 2006.

[25] C. Presilla, J. Sjöstrand. Transport properties in resonant tunneling heterostructures. J. Math. Phys., 37(10), 4816-4844, 1996.

[26] C. Presilla, J. Sjöstrand. Nonlinear resonant tunneling in systems coupled to quantum reservoirs. Phys. Rev. B: Condensed matter, 55 no15, 9310-9313, 1997. 
[27] M. Reed, B. Simon. Methods of modern mathematical physics vol. IV: Analysis of Operators. Academic Press, New YorK, 1978.

[28] V.A. Ryzhov. On a singular and an absolutely continuous subspace of a nonselfadjoint operator whose characteristic function has boundary values on the real axis. Funct. Anal. Appl. 32, no. 3, 208-212, 1998.

[29] V.A. Ryzhov. Equipped absolutely continuous subspaces and stationary construction of the wave operators in the non-self-adjoint scattering theory. J. Math. Sci., 85, no. 2, 1997.

[30] V.A. Ryzhov, Functional model of a class of non-selfadjoint extensions of symmetric operators. Oper. Theory Adv. Appl., vol. 174, 117-158, 2007.

[31] D.R. Yafaev. Mathematical Scattering Theory: Analytic theory. Mathematical Surveys and Monographs vol. 158, American Mathematical Society, Providence, 2010.

[32] D.R. Yafaev. Mathematical Scattering Theory: General Theory. American Mathematical Society, Providence, 1992. 